\title{
Additive inhomogeneous Diophantine inequalities
}

\author{
by \\ D. Eric Freeman (Boulder, CO, and Princeton, NJ)
}

1. Introduction. In the study of Diophantine equations and inequalities, most results concern homogeneous polynomials. For example, suppose that $G(\mathbf{x})$ is a homogeneous polynomial, of odd degree $k$ in $s$ variables, with real coefficients. Schmidt [16] has given the impressive result that there exists a positive integer $s_{0}(k)$, which depends only on the degree $k$, so that if $s \geq s_{0}(k)$, then there is a vector $\mathbf{x} \in \mathbb{Z}^{s} \backslash\{\mathbf{0}\}$ satisfying the inequality

$$
|G(\mathbf{x})|<1 .
$$

In other words, if there are enough variables, in terms of the degree only, then there is a non-trivial solution of the Diophantine inequality (1). Earlier, Birch [4] had proved a similar theorem for Diophantine equations. But there are still very few known results about inhomogeneous polynomials.

For Diophantine equations, this is perhaps to be expected, for reasons more convincing than simply because homogeneous polynomials have a nicer form. For if one considers equations such as

$$
(G(\mathbf{x}))^{3}-2=0,
$$

where $G(\mathbf{x})$ is an integral polynomial, we can see that there is good reason to restrict to the homogeneous case: the number of variables $s$ here can be chosen as large as we like, and $G(\mathbf{x})$ can even be chosen so that the equation (2) has real solutions, yet there are clearly no integral solutions of (2).

Diophantine equations are specific cases of Diophantine inequalities, so there is still cause to be careful in the inequality case. Moreover, if $F(\mathbf{x})$ is a real multiple of an integral form, then the inequality $|F(\mathbf{x})|<\varepsilon$ reduces to the equation $F(\mathbf{x})=0$, for sufficiently small $\varepsilon$. Now consider the Diophantine inequality $|F(\mathbf{x})|<\varepsilon$ in the alternative case, when $F$ is not a real multiple of an integral form, or equivalently, when the coefficients of $F$ are not all in

2000 Mathematics Subject Classification: Primary 11D75; Secondary 11D41, 11D72, $11 \mathrm{P} 55$.

Key words and phrases: Diophantine inequalities, inhomogeneous polynomials, forms in many variables, applications of the Hardy-Littlewood method.

Supported by a National Science Foundation Postdoctoral Fellowship. 
rational ratio. Perhaps one can more often solve Diophantine inequalities in such cases.

As a natural first step towards considering such inhomogeneous inequalities, we look at inhomogeneous polynomials which are themselves sums of polynomials in one variable. Thus suppose that $\kappa$ is a real number and that $h_{1}(y), \ldots, h_{s}(y)$ are real polynomials, each in one variable. We look for integral solutions $\left(y_{1}, \ldots, y_{s}\right)$ to Diophantine inequalities of the type

$$
\left|h_{1}\left(y_{1}\right)+\ldots+h_{s}\left(y_{s}\right)-\kappa\right|<\varepsilon .
$$

We give two definitions so that we may state our result more easily.

Definition. Suppose that $k$ and $s$ are positive integers. Also, for $1 \leq i \leq s$, suppose that $h_{i}(y)$ is a polynomial with real coefficients, given by

$$
h_{i}(y)=\beta_{i k} y^{k}+\beta_{i(k-1)} y^{k-1}+\ldots+\beta_{i 1} y+\beta_{i 0} .
$$

Then we say that the polynomials $h_{1}, \ldots, h_{s}$ satisfy the irrationality condition if there exist integers $i_{1}$ and $i_{2}$ with $1 \leq i_{1}, i_{2} \leq s$ and integers $j_{1}$ and $j_{2}$ with $1 \leq j_{1}, j_{2} \leq k$ for which one has

$$
\frac{\beta_{i_{1} j_{1}}}{\beta_{i_{2} j_{2}}} \notin \mathbb{Q} \text {. }
$$

Of course here we assume that $\beta_{i_{2} j_{2}}$ is non-zero.

This condition will serve to guarantee that one of the ratios of the coefficients of the sum polynomial $h_{1}\left(y_{1}\right)+\ldots+h_{s}\left(y_{s}\right)$ is irrational, and to ensure that we are not essentially considering a Diophantine equation. We make a few observations. Note that we could have $i_{1}=i_{2}$, so that the coefficients whose ratio is irrational could come from only one polynomial. Another observation, which is very important, is that we require $j_{1} \geq 1$ and $j_{2} \geq 1$. In other words, neither of the coefficients whose ratio is irrational is one of the constant terms. To see why we make such a requirement, consider the simple inequality

$$
\left|y_{1}^{3}+\ldots+y_{s-1}^{3}+\left(y_{s}^{3}+\pi\right)\right|<\varepsilon .
$$

This is an inequality of the form (3) with $\kappa=0$ and with $h_{s}\left(y_{s}\right)=y_{s}^{3}+\pi$. For $\varepsilon \leq 1 / 10$, say, this inequality has no solutions in integers $y_{1}, \ldots, y_{s}$, even though there is clearly an irrational ratio among the coefficients. The problem here is that we are essentially still dealing with an integral polynomial in this case. We give one more definition.

Definition. Suppose that $H(\mathbf{x})$ is a sum of non-constant polynomials $h_{i}\left(x_{i}\right)$, each of degree at most $k$, where $k$ is a positive integer. Then we say that $H(\mathbf{x})$ is an indefinite polynomial if not all of the leading coefficients of the polynomials $h_{i}$ are of the same sign, or if any of the polynomials $h_{i}$ has odd degree. We say that $H(\mathbf{x})$ is a positive-definite polynomial (respectively, 
negative-definite polynomial) if all of the leading coefficients of the polynomials $h_{i}$ are positive (respectively, negative) and all of the polynomials $h_{i}$ are of even degree.

We note that a definition of a somewhat similar nature has been given by Cook and Raghavan [7].

Having given the above definitions, we may now state our main result.

THEOREM 1. Suppose that $k$ is a positive integer. Then there is a positive integer $s_{0}(k)$ such that any integer $s$ with $s \geq s_{0}(k)$ has the following property:

Suppose that, for $1 \leq i \leq s$, the polynomial $h_{i}(y)$ has real coefficients, is non-constant and is of degree at most $k$, and that the polynomials $h_{1}, \ldots, h_{s}$ satisfy the irrationality condition. Fix a positive number $\varepsilon$ and any real number $\kappa$. Set

$$
H(\mathbf{y})=H\left(y_{1}, \ldots, y_{s}\right)=\sum_{i=1}^{s} h_{i}\left(y_{i}\right) .
$$

Finally, suppose that $H(\mathbf{y})$ is an indefinite polynomial. Then there exist infinitely many s-tuples of integers $\mathbf{z}=\left(z_{1}, \ldots, z_{s}\right)$ for which

$$
|H(\mathbf{z})-\kappa|<\varepsilon \text {. }
$$

Moreover, we in fact may take $s_{0}(k)$ to satisfy

$$
s_{0}(k) \sim 4 k \log k .
$$

Note that, for example, Theorem 1 states that the values taken at integer points by a sum of $s_{0}(k)$ polynomials, which are of odd degree $k$ and satisfy the irrationality condition, are dense on the real line.

For sums of general polynomials, Theorem 1 is one of the first results of its kind. However, the theorem is essentially already known in the special case in which every polynomial $h_{i}$ takes the simple form $h_{i}\left(y_{i}\right)=\beta_{i k} y_{i}^{k}$. (See the paper of Brüdern and Cook [6]. They only prove the theorem in this special case for $\kappa=0$, but their argument extends to prove this special case for all $\kappa$.) In fact, in this case, one can take $s_{0}(k)$ asymptotic to $k \log k$ by combining the smooth number methods of Wooley with the work in [6]. Currently, we cannot use these methods for general polynomials however, so we need the constant 4 here. We also note that we could follow our proof carefully and give an error term of order $k$ in the asymptotic formula for $s_{0}(k)$, and we could also likely fine-tune our method to give better bounds of this type, but we choose not to, for ease of exposition. Such techniques would not allow us to reduce the constant factor 4 at any rate, without some new ideas. 
Before stating another result, we must give some notation and definitions. For real vectors $\mathbf{x} \in \mathbb{R}^{s}$, we define

$$
|\mathbf{x}|=\max _{1 \leq i \leq s}\left|x_{i}\right| .
$$

Also, as is usual, by a non-trivial solution $\mathbf{x}$ of an inequality we mean that $\mathbf{x}$ is a solution which is not the zero vector.

We give a more technical version of the above result, which in fact implies Theorem 1. In Theorem 1, we only considered polynomials which were indefinite. Now we consider both definite and indefinite polynomials.

THEOREM 2. Suppose that $k$ is a positive integer. Then there is a positive integer $s_{0}(k)$ such that any integer $s$ with $s \geq s_{0}(k)$ has the following property:

Suppose that, for $1 \leq i \leq s$, the polynomial

$$
h_{i}(y)=\beta_{i k} y^{k}+\beta_{i(k-1)} y^{k-1}+\ldots+\beta_{i 1} y+\beta_{i 0}
$$

has real coefficients, is non-constant, and is of degree at most $k$, and that $h_{1}, \ldots, h_{s}$ satisfy the irrationality condition. Set

$$
H(\mathbf{y})=H\left(y_{1}, \ldots, y_{s}\right)=\sum_{i=1}^{s} h_{i}\left(y_{i}\right) .
$$

Now fix a positive number $\varepsilon$. Then there are positive constants $C_{1}, C_{2}$ and $C_{3}$ with $C_{2}<C_{3}$, which depend only on $k$ and the coefficients $\beta_{i j}$, such that, given any positive number $P$ which is sufficiently large in terms of $k$ and $\varepsilon$ and the coefficients $\beta_{i j}$, the following two statements hold:

(i) Suppose that $H(\mathbf{y})$ is an indefinite polynomial. If $M$ is a real number with $|M| \leq C_{1} P$, then there exists a non-trivial s-tuple of integers $\mathbf{z}=$ $\left(z_{1}, \ldots, z_{s}\right)$ with $|\mathbf{z}| \leq P$ for which

$$
|H(\mathbf{z})-M|<\varepsilon \text {. }
$$

(ii) If $H(\mathbf{y})$ is a positive-definite polynomial and if $M$ is a real number with $C_{2} P \leq M \leq C_{3} P$, then there exists an s-tuple of integers $\mathbf{z}=$ $\left(z_{1}, \ldots, z_{s}\right)$ with $|\overline{\mathbf{z}}| \leq P$ for which

$$
|H(\mathbf{z})-M|<\varepsilon \text {. }
$$

Moreover, we in fact may take $s_{0}(k)$ to satisfy

$$
s_{0}(k) \sim 4 k \log k .
$$

We observe that we also of course have a result similar to case (ii) of this theorem if $H(\mathbf{x})$ is a negative-definite polynomial where we instead assume that $-C_{3} P \leq M \leq-C_{2} P$ holds. Such a result can of course be obtained by applying case (ii) of this theorem to $-H(\mathbf{x})$. We also note that if we assumed that each of the polynomials $h_{i}$ were of degree $k$, then we could take the 
solutions $\mathbf{z}$ in cases (i) and (ii) to satisfy $|\mathbf{z}| \leq P^{1 / k}$ instead of $|\mathbf{z}| \leq P$. We note as well that we could give some sort of bounds for the constants $C_{1}$, $C_{2}$ and $C_{3}$. However, in our proof, we focus on keeping the number $s_{0}(k)$ of variables necessary from being too large, and this comes at the price of any chance of determining the best possible constants here, so we do not concern ourselves with such bounds.

We also note, as Professor Schmidt observed, that in case (ii) one can show by a straightforward argument that we can assume only that $M$ satisfies $M \geq C_{4}$ for some positive constant $C_{4}$, if one allows $C_{4}$ to depend on $k$, the coefficients $\beta_{i j}$, and $\varepsilon$.

We turn now to considering some related results. We do not mention any more results on Diophantine inequalities involving homogeneous polynomials, having already mentioned the work of Schmidt [16] above. We now consider instead results about Diophantine inequalities which involve inhomogeneous polynomials. First, there is the recent work of Bentkus and Götze. Let $k$ be an even integer and suppose that $s$ satisfies $s \geq s_{1}(k)$, where $s_{1}(k)$ is a function they give which satisfies $s_{1}(k) \ll k^{4} 4^{k}$. Then let

$$
F(\mathbf{x})=\lambda_{1} x_{1}^{k}+\ldots+\lambda_{s} x_{s}^{k}+R(\mathbf{x}),
$$

where $R(\mathbf{x})$ is a polynomial in $\mathbf{x}=\left(x_{1}, \ldots, x_{s}\right)$ of degree strictly less than $k$. Suppose that $\lambda_{i}>0$ for $1 \leq i \leq s$. Also, suppose that for some $1 \leq i<j \leq s$, we have $\lambda_{i} / \lambda_{j} \notin \mathbb{Q}$. Next, fix a positive number $\varepsilon$. Then for any positive number $M$ which is sufficiently large in terms of $k$ and $s$ and $\varepsilon$ and the coefficients of $F(\mathbf{x})$, they prove that there is a solution of

$$
|F(\mathbf{x})-M|<\varepsilon .
$$

(See [3]. In fact, they give a much stronger result concerning the distribution of the values of $F(\mathbf{x})$.) This is one of the first forays into the study of inhomogeneous Diophantine inequalities. And indeed, as they remark in their paper, their methods can most likely be used to non-trivially solve inequalities of the type $|F(\mathbf{x})|<\varepsilon$, where $F(\mathbf{x})$ is as above but is indefinite in nature.

There are fundamental differences between their result and ours, and also in the methods used. The polynomial $F(\mathbf{x})$ is of course of a more general type than $H(\mathbf{x})$, the sum of polynomials which we consider. However, if one restricts $F(\mathbf{x})$ to be a sum of polynomials, the work of Bentkus and Götze requires one to assume that there is an irrational ratio among the coefficients of the highest degree terms of these polynomials, rather than our more relaxed assumption that there is an irrational ratio among the coefficients which are not constant terms. Of course, they require many more variables for large $k$, but this is hardly surprising concerning the more general form of the polynomial $F(\mathbf{x})$. 
Most other known results about Diophantine inequalities involving inhomogeneous polynomials concern sums of constant multiples of mixed powers. We consider, for example, the following result due to Brüdern [5], which we state in a simplified form. Suppose that $\lambda_{1}, \ldots, \lambda_{6}$ are non-zero real numbers such that at least one of the ratios $\lambda_{i} / \lambda_{j}$ is irrational. Then for any real number $\varepsilon>0$ and any real number $\mu$, there are integers $x_{1}, \ldots, x_{6}$, not all zero, such that one has

$$
\left|\lambda_{1} x_{1}^{2}+\lambda_{2} x_{2}^{3}+\lambda_{3} x_{3}^{3}+\lambda_{4} x_{4}^{3}+\lambda_{5} x_{5}^{3}+\lambda_{6} x_{6}^{3}-\mu\right|<\varepsilon .
$$

There are other results of a similar nature. (See for example [11], or see also [5] for references to other such results.) Finally, we mention that there are a few other sporadic results which concern inhomogeneous Diophantine inequalities. We direct the reader to the work of Cook and Raghavan [7] and the work of Watson [19], both concerning inequalities involving quadratic polynomials.

There has also been some work on inhomogeneous Diophantine equations. Let $f(x)$ be a polynomial of degree $k$ with integer coefficients which satisfies the property that if $d$ is a positive integer which divides $f(x)$ for all integers $x$, then $d=1$. Kamke [14] showed in 1921 that there is an integer $s$, depending on the polynomial $f$, such that for sufficiently large $n$ there is an integer solution of the equation

$$
f\left(x_{1}\right)+\ldots+f\left(x_{s}\right)=n .
$$

See the work of Wooley ([23], Theorem 9), Ford [12] and Nathanson ([15], Sections 11.4 and 12.4) for recent work on this type of problem and references to earlier results.

Now suppose that $F(\mathbf{x})$ is a general polynomial, not necessarily homogeneous, of degree $k$ in $s$ variables. Then we call the sum of the terms of degree 3 the cubic part of the polynomial, and we refer to the sum of the terms of degree 2 as the quadratic part. Watson [20] has given a result about quadratic integral polynomials whose quadratic part is a positivedefinite quadratic form, in particular concerning the values the polynomials take at integer points, under certain congruence conditions. Davenport and Lewis [9] have given conditions under which one can solve the Diophantine equation $C(\mathbf{x})=0$, where $C(\mathbf{x})$ is a cubic integral polynomial. They require $C(\mathbf{x})$ to satisfy certain congruence conditions, and require certain algebraic restrictions on the cubic part of $C(\mathbf{x})$.

We briefly discuss the methods we use to prove Theorems 1 and 2 . We use the Davenport-Heilbronn method, with variations based on the ideas of Bentkus and Götze [2]. We note that we need not use the ideas of Bentkus and Götze to attack the case in which $H(\mathbf{x})$ is an indefinite polynomial. However, we use the method since it also gives us a result in the case in 
which $H(\mathbf{x})$ is positive-definite. We note that it also allows us to obtain an asymptotic lower bound of a certain kind for the number of solutions in a box of size $P$ for every large positive number $P$, which the usual DavenportHeilbronn method does not yield.

As in most applications of the Davenport-Heilbronn method, there are two key ingredients: an analogue of Hua's inequality and an analogue of Weyl's inequality. To obtain our analogue of Hua's inequality, we use a diminishing ranges argument and bounds essentially due to Vinogradov, and some ideas of Davenport and Roth [10]. We must take more care, however, because of the nature of the techniques of Bentkus and Götze. Often, when using the Hardy-Littlewood circle method or the DavenportHeilbronn method, one considers an exponential sum $T(\alpha)$ of length $P$, say. Then an analogue of Hua's inequality is usually of the form

$$
\int_{0}^{1}|T(\alpha)|^{s} d \alpha \ll P^{s-k+\eta},
$$

where $\eta$ can be taken to be any positive number. When using the techniques of Bentkus and Götze, one instead must be able to replace the right hand side above by simply $P^{s-k}$. We call such a bound an "exact Hua inequality", as often this is the best possible bound (up to a constant factor) that one can expect. We obtain our exact Hua inequality by first proving a more typical analogue of Hua's inequality, and then applying the Hardy-Littlewood method with some mild twists. Here we also use some techniques of Baker, Hua and Vaughan.

To obtain our analogue of Weyl's inequality, we must also do a bit more because we have chosen to use the techniques of Bentkus and Götze. In fact, as remarked above, it would be possible (in the indefinite case) to not use these techniques; to do so, we would employ a result of Baker (Theorem 5.1 of [1]) as our analogue of Weyl's inequality. However, as with most analogues of (technically, the contrapositive of) Weyl's inequality, Baker's result states that if an exponential sum $T(\alpha)$ is large, then one has good rational approximations to $\alpha$, but where the quality of these rational approximations is described by bounds which have a factor of $P^{\varepsilon}$ in them. We cannot use such results because of these factors. When we have similar results without such factors, we call them "exact Weyl inequalities". In a previous version of the paper, we developed work from [13], using techniques of Schmidt [17], in order to obtain such an exact Weyl inequality. However, Professor Wooley has pointed out a much more straightforward proof, which we give below. This simplifies the proof to a very large extent.

Finally, we note that we could also take $s_{0}(k)=k 2^{k-1}+1$ in Theorems 1 and 2 , and in this case we could even find an asymptotic lower bound of 
the expected order of magnitude for the number of solutions of our inequality. We briefly note that to do so, one would use a largely similar method but without the diminishing ranges, and one would give some alterations to obtain a different version of Lemma 5. To obtain the required version of Lemma 5, one would only need to treat differently the version of the minor arcs given in the proof of Lemma 5. This may be done by using a slight modification of the result of Baker mentioned above. (See Theorem 5.1 of $[1]$.)

I would like to take this opportunity to thank Professor Schmidt for a conversation during which this question arose. I would also like to thank Professor Wooley for making an important observation which led to a considerable simplification of the proof of Lemma 6 . I am grateful to him as well for pointing out that my original results, in which all of the polynomials $h_{i}$ were of degree $k$, could be readily extended to yield the current result.

2. A proposition. In this section, we give a technical proposition which implies Theorems 1 and 2. In fact, it will be enough to show that the proposition implies Theorem 2, as we now demonstrate by proving that Theorem 2 implies Theorem 1. This is clear, except for the infinitude of solutions of the inequality (4). Even this part is fairly straightforward, but we prove it for completeness.

To see this, fix a real number $\kappa$ and a positive number $\varepsilon$. We construct a sequence of distinct integral solutions $\mathbf{z}_{n}$ of (4) as follows. For positive integers $n$, let

$$
M_{n}=\kappa+\varepsilon\left(1-2 / 4^{n}\right) \text { and } \varepsilon_{n}=\varepsilon / 4^{n} .
$$

For each $n \in \mathbb{Z}^{+}$, we apply Theorem 2 with $P$ large enough in terms of $\varepsilon, k$ and the coefficients $\beta_{i j}$ and with $\left|M_{n}\right| \leq C_{1} P$. In this manner, we have $\mathbf{z}_{n} \in \mathbb{Z}^{s}$ with

$$
\left|H\left(\mathbf{z}_{n}\right)-M_{n}\right|<\varepsilon_{n} .
$$

Observe from (7) and (8) that

$$
H\left(\mathbf{z}_{n-1}\right)<\kappa+\varepsilon\left(1-1 / 4^{n-1}\right)<\kappa+\varepsilon\left(1-3 / 4^{n}\right)<H\left(\mathbf{z}_{n}\right)
$$

and

$$
\kappa-\varepsilon \leq \kappa+\varepsilon\left(1-3 / 4^{n}\right)<H\left(\mathbf{z}_{n}\right)<\kappa+\varepsilon\left(1-1 / 4^{n}\right) \leq \kappa+\varepsilon .
$$

By (9), the vectors $\mathbf{z}_{n}$ are distinct, and by (10), the vectors $\mathbf{z}_{n}$ are solutions of (4). Thus Theorem 2 implies Theorem 1.

We now state the proposition which implies Theorems 1 and 2. In this section, we demonstrate how the proposition implies Theorem 2. The remainder of the paper is dedicated to the proof of the proposition. 
Proposition 1. Fix a positive number $\varepsilon$. Suppose that $k$ is an integer satisfying $k \geq 2$. Define

$$
\phi=\phi(k)=\left(8 k^{2}\left(\log k+\frac{1}{2} \log \log k+2\right)\right)^{-1} .
$$

Also, for convenience, set $m=m(k)=2 k+4$. Define $t$ to be the smallest positive integer which satisfies

$$
(1-1 / k)^{t}<(2 k+1) \phi / k .
$$

Now, for $1 \leq i \leq 2 t$ and $1 \leq n \leq m$, suppose that $v_{i}$ and $w_{n}$ are integers satisfying $2 \leq v_{i}, w_{n} \leq k$. As well, for $1 \leq i \leq 2 t$ and $1 \leq n \leq m$, suppose that the polynomials

$$
\begin{aligned}
& f_{i}(x)=\lambda_{i v_{i}} x^{v_{i}}+\lambda_{i\left(v_{i}-1\right)} x^{v_{i}-1}+\ldots+\lambda_{i 1} x, \\
& g_{n}(y)=\mu_{n w_{n}} y^{w_{n}}+\mu_{n\left(w_{n}-1\right)} y^{w_{n}-1}+\ldots+\mu_{n 1} y
\end{aligned}
$$

have real coefficients, and are of degree $v_{i}$ and $w_{n}$, respectively. Assume that for every $j$ with $2 \leq j \leq k$, there are an even number of the degrees $v_{i}$ which equal $j$. Also assume that for some $j_{1}$ and $j_{2}$ with $1 \leq j_{1} \leq w_{1}$ and $1 \leq j_{2} \leq w_{2}$, we have

$$
\mu_{1 j_{1}} / \mu_{2 j_{2}} \notin \mathbb{Q},
$$

where of course here we assume that $\mu_{2 j_{2}}$ is non-zero. Suppose as well that $P$ is a positive number which is sufficiently large in terms of $k$ and $\varepsilon$ and the coefficients $\lambda_{i j}$ and $\mu_{n j}$. Consider the inequality

$$
\left|f_{1}\left(x_{1}\right)+\ldots+f_{2 t}\left(x_{2 t}\right)+g_{1}\left(y_{1}\right)+\ldots+g_{m}\left(y_{m}\right)-M\right|<\varepsilon .
$$

The following two statements hold.

(i) Suppose that $\mu_{1 w_{1}}>0$ and $\mu_{2 w_{2}}<0$. Suppose in addition that $1 \leq\left|\mu_{1 w_{1}} / \mu_{2 w_{2}}\right| \leq 2$. Also suppose that

$$
\sum_{i=1}^{2 t} \sum_{j=1}^{v_{i}}\left|\lambda_{i j}\right|+\sum_{n=3}^{m} \sum_{j=1}^{w_{n}}\left|\mu_{n j}\right| \leq \frac{\mu_{1 w_{1}}}{2^{k+3}} .
$$

Finally, suppose that $M$ is a real number with

$$
|M| \leq \mu_{1 w_{1}} P / 8 \text {. }
$$

Then there exists a non-trivial $(2 t+m)$-tuple of integers

$$
(\mathbf{x}, \mathbf{y})=\left(x_{1}, \ldots, x_{2 t}, y_{1}, \ldots, y_{m}\right) \quad \text { with }|\mathbf{x}|,|\mathbf{y}| \leq 2 P
$$

such that (13) holds.

(ii) Suppose that $\mu_{1 w_{1}}>0$ for $1 \leq i \leq s$ and that $M$ is a real number with

$$
\mu_{1 w_{1}} P / 4 \leq M \leq 3 \mu_{1 w_{1}} P / 4 .
$$


Additionally, suppose that

$$
\sum_{i=1}^{2 t} \sum_{j=1}^{v_{i}}\left|\lambda_{i j}\right|+\sum_{n=2}^{m} \sum_{j=1}^{w_{n}}\left|\mu_{n j}\right| \leq \frac{\mu_{1 w_{1}}}{2^{k+3}} .
$$

Then there exists a non-trivial $(2 t+m)$-tuple of integers

$$
(\mathbf{x}, \mathbf{y})=\left(x_{1}, \ldots, x_{2 t}, y_{1}, \ldots, y_{m}\right) \quad \text { with }|\mathbf{x}|,|\mathbf{y}| \leq 2 P
$$

such that (13) holds.

We note that the numerical constants appearing in conditions (14)-(17) could all doubtlessly be improved, but we do not concern ourselves with the optimal choices for these constants.

We show now that Proposition 1 implies Theorem 2. Note first of all that it is enough to prove Theorem 2 in the case in which the coefficients $\beta_{i 0}$ are all zero. For suppose that we have established the theorem in this special case. We may choose a positive constant $C_{1}^{\prime}$, for $P$ large in terms of the coefficients $\beta_{i 0}$, such that $|M| \leq C_{1}^{\prime} P$ implies that the required condition $\left|M-\sum_{i=1}^{s} \beta_{i 0}\right| \leq C_{1} P$ holds. Thus case (i) of Theorem 2 , in the special case in which the coefficients are all zero, implies case (i) of Theorem 2 in the general case, albeit with a different constant $C_{1}$. In a similar manner, we may deduce case (ii) of Theorem 2 in general, if we have established it in the special case in which all of the coefficients $\beta_{i 0}$ are zero. Thus, in what remains, we assume that all of these constant terms are zero.

Now observe that Theorem 2 holds in the case in which at least one of the polynomials $h_{i}$ has degree one. To see this, it is enough to show that the theorem holds for a sum of polynomials $\beta_{11} y_{1}+h_{2}\left(y_{2}\right)$, where $\beta_{11} y_{1}$ and $h_{2}$ satisfy the irrationality condition, as one may set the variables corresponding to all other polynomials equal to zero, while ensuring that the irrationality condition still holds; after all, the ratios of $\beta_{11}$ to the other (non-constant term) coefficients cannot all be rational. It follows from the irrationality condition that $g_{2}\left(y_{2}\right)=h_{2}\left(y_{2}\right) / \beta_{11}$ has at least one irrational coefficient. By a famous result of Weyl [21], the values of $g_{2}\left(y_{2}\right)$ are uniformly distributed modulo one for integers $y_{2}$. Thus for sufficiently large $P$, and $M$ with $M \leq\left(\max \left(C_{1}, C_{3}\right)\right) P$, we may find an integer $y_{2}$ with $\left|y_{2}\right| \leq c_{0} P$, for some positive constant $c_{0}$, such that

$$
\left\|g_{2}\left(y_{2}\right)-M / \beta_{11}\right\|<\varepsilon /\left|\beta_{11}\right|
$$

here, as usual, we denote the nearest integer to a real number $x$ by $\|x\|$. For sufficiently small choices of $c_{0}, C_{1}$ and $C_{3}$, we may choose $\left|y_{1}\right| \leq P$ so that

$$
\left|y_{1}+g_{2}\left(y_{2}\right)-M / \beta_{11}\right|<\varepsilon /\left|\beta_{11}\right| \text {. }
$$

Clearly we then have $\left|\beta_{11} y_{1}+h_{2}\left(y_{2}\right)-M\right|<\varepsilon$. Observe that in this special case, which includes the case $k=1$ of the theorem, we could take $s_{0}(k)=2$. 
We now turn to the deduction of Theorem 2 in the special case in which all of the polynomials $h_{i}$ have degree at least 2 , and, as remarked above, in which all of their constant terms are zero.

We first observe that, for $k \geq 2$, there exists a function $s_{0}(k)$ for which

$$
2 t+m \leq s_{0}(k) \text { and } s_{0}(k) \sim 4 k \log k .
$$

Although this may be seen fairly readily, we give a simple elementary proof for completeness. Observe from calculus that $1-x \leq e^{-x}$ for all real numbers $x$, and thus for all real numbers $y$ with $y \geq 1$, we have

$$
(1-1 / y)^{y} \leq 1 / e .
$$

For any real number $C^{\prime}$ with $C^{\prime} \geq 1$, it follows that if we set

$$
w=k\left(2 \log k+\log C^{\prime}+\log \log k\right),
$$

we have $(1-1 / k)^{w} \leq\left(C^{\prime} k^{2} \log k\right)^{-1}$. Thus for a sufficiently large number $C^{\prime}$, we can see that $w$ satisfies

$$
(1-1 / k)^{w}<(2 k+1) \phi / k .
$$

Now let $t^{\prime}=\lceil w\rceil$. Note that $w \sim 2 k \log k$, whence we certainly have $t^{\prime} \sim$ $2 k \log k$. But recall that $t$ is the least integer for which (12) holds, whence $t \leq t^{\prime}$. Recalling as well that $m=2 k+4$, we see that we may take $2 t+m \leq$ $s_{0}(k)$, where $s_{0}(k) \sim 4 k \log k$. We now assume that $s$ is an integer which satisfies $s \geq s_{0}(k)$, and thus

$$
s \geq 2 t+m \text {. }
$$

Now, for $1 \leq i \leq s$, suppose that the polynomial $h_{i}$ is of degree $d_{i}$, where $2 \leq d_{i} \leq k$. Note that therefore $\beta_{i d_{i}}$ is non-zero for $1 \leq i \leq s$. The polynomials $h_{1}, \ldots, h_{s}$ satisfy the irrationality condition, so there exist $i_{1}$, $i_{2}, j_{1}$ and $j_{2}$ with $1 \leq i_{1}, i_{2} \leq s$ and $1 \leq j_{1} \leq d_{i_{1}}$ and $1 \leq j_{2} \leq d_{i_{2}}$ such that $\beta_{i_{1} j_{1}} / \beta_{i_{2} j_{2}}$ is irrational. Suppose that $i_{1}=i_{2}$. Fix some $i_{0}$ with $i_{0} \neq i_{1}$ and $1 \leq i_{0} \leq s$. Then at least one of $\beta_{i_{1} j_{1}} / \beta_{i_{0} d_{i_{0}}}$ and $\beta_{i_{1} j_{2}} / \beta_{i_{0} d_{i_{0}}}$ must be irrational. In this manner, we can assume that $i_{1} \neq i_{2}$.

Now suppose that we are in the setting of case (i) of Theorem 2, so that $H(\mathbf{y})$ is an indefinite polynomial. We claim that there exists $i_{0}$ with $1 \leq i_{0} \leq s$ for which $\beta_{i_{1} d_{i_{1}}} / \beta_{i_{0} d_{i_{0}}}$ is negative. In the case in which $d_{i}$ is even for $1 \leq i \leq s$, this is clear, as not all of the coefficients $\beta_{i d_{i}}$ can have the same sign. If there exists some $i_{0}$ for which $d_{i_{0}}$ is odd, then by replacing $z_{i_{0}}$ by $-z_{i_{0}}$ if necessary, we may assume that $\beta_{i_{1} d_{i_{1}}} / \beta_{i_{0} d_{i_{0}}}$ is negative. Fix this $i_{0}$. Observe that we must have $i_{0} \neq i_{1}$.

Now assume as well that $\beta_{i_{1} d_{i_{1}}} / \beta_{i_{2} d_{i_{2}}}$ is positive; note in particular that $i_{0}$ then must be distinct from both $i_{1}$ and $i_{2}$. In this case, both $\beta_{i_{1} d_{i_{1}}} / \beta_{i_{0} d_{i_{0}}}$ and $\beta_{i_{2} d_{i_{2}}} / \beta_{i_{0} d_{i_{0}}}$ are negative, while at least one of $\beta_{i_{1} j_{1}} / \beta_{i_{0} d_{i_{0}}}$ and $\beta_{i_{2} j_{2}} / \beta_{i_{0} d_{i_{0}}}$ is irrational. So without loss of generality, in case (i) of Theorem 2 , we may 
assume that $\beta_{i_{1} j_{1}} / \beta_{i_{2} j_{2}}$ is irrational and that $\beta_{i_{1} d_{i_{1}}} / \beta_{i_{2} d_{i_{2}}}$ is negative. By relabeling variables, we may assume that both $\beta_{(2 t+1) j_{1}} / \beta_{(2 t+2) j_{2}} \notin \mathbb{Q}$ for some $j_{1}$ and $j_{2}$ with $1 \leq j_{1} \leq d_{j_{1}}$ and $1 \leq j_{2} \leq d_{j_{2}}$ and also, in case (i) of Theorem 2, that $\beta_{(2 t+1) d_{2 t+1}}>0$ and $\beta_{(2 t+2) d_{2 t+2}}<0$.

Recall now that by (18) and the definition of $m$, we have $s \geq 2 t+2 k+4$. We will now relabel the polynomials $h_{1}, \ldots, h_{2 t}$ so that we may assume that, for each $j$ with $2 \leq j \leq k$, there are an even number of polynomials $h_{i}$ among $h_{1}, \ldots, h_{2 t}$ of degree $j$. To do so, we consider each $j$ with $2 \leq j \leq k$. We start with $j=2$, and work our way up to $j=k$. If there is an even number of polynomials of degree $j$ among $h_{1}, \ldots, h_{2 t}$, we proceed and consider the polynomials of degree $j+1$. Now suppose that there is an odd number of polynomials of degree $j$ among $h_{1}, \ldots, h_{2 t}$. As $2 t$ is of course even, there is another degree $j^{\prime}$ such that there is an odd number of polynomials of degree $j^{\prime}$ among $h_{1}, \ldots, h_{2 t}$. Let one of these polynomials of degree $j^{\prime}$ be $h_{i_{j^{\prime}}}$, say. If there is some polynomial of degree $j$ among $h_{2 t+3}, h_{2 t+4}, \ldots, h_{s}$, say $h_{i_{j}}$, then we switch the indices of $h_{i_{j^{\prime}}}$ and $h_{i_{j}}$. In so doing, we are left with an even number of polynomials of both degrees $j^{\prime}$ and $j$ among the polynomials $h_{1}, \ldots, h_{2 t}$. Now, alternatively, suppose that there is an odd number of polynomials of degree $j$ among $h_{1}, \ldots, h_{2 t}$ but that none of the polynomials $h_{2 t+3}, \ldots, h_{s}$ have degree $j$. Then, as $s \geq 2 t+2 k+4$, there is certainly at least one degree $j^{\prime \prime}$ with $2 \leq j^{\prime \prime} \leq k$ for which there are at least two polynomials $h_{i}$ of degree $j^{\prime \prime}$ among $h_{2 t+3}, \ldots, h_{s}$. We exchange these two polynomials with a polynomial of degree $j$ and a polynomial of degree $j^{\prime}$, each among $h_{1}, \ldots, h_{2 t}$, where $j^{\prime} \neq j$ is such that there is an odd number of polynomials of degree $j^{\prime}$ among $h_{1}, \ldots, h_{2 t}$. Then among $h_{1}, \ldots, h_{2 t}$, we are left with an even number of polynomials of degrees $j$ and $j^{\prime}$, and the parity of the number of polynomials of degree $j^{\prime \prime}$ is unchanged. Now we may proceed to consider the polynomials of degree $j+1$, repeating this step until we reach the degree $j=k$. In this manner, we are left with an even number of polynomials of every degree $j$ with $2 \leq j \leq k$, among the polynomials $h_{1}, \ldots, h_{2 t}$.

We now define sets of polynomials $f_{1}, \ldots, f_{2 t}$ and $g_{1}, \ldots, g_{m}$ such that if

$$
\left|f_{1}\left(x_{1}\right)+\ldots+f_{2 t}\left(x_{2 t}\right)+g_{1}\left(y_{1}\right)+\ldots+g_{m}\left(y_{m}\right)-M\right|<\varepsilon
$$

has an integral solution $(\mathbf{x}, \mathbf{y})$, then there is a corresponding integral solution of

$$
|H(\mathbf{z})-M|<\varepsilon .
$$

We will then apply Proposition 1 to the inequality (19), and thus show that (20) has an integral solution. Our polynomials $f_{i}$ and $g_{n}$ are similar in both cases (i) and (ii) of Theorem 2, although with slight differences.

Now recall that the polynomials $h_{i}$ are of degree $d_{i}$ for $1 \leq i \leq s$. We define 


$$
v_{i}=d_{i} \quad \text { for } 1 \leq i \leq 2 t \quad \text { and } \quad w_{n}=d_{2 t+n} \quad \text { for } 1 \leq n \leq m .
$$

In both cases (i) and (ii) of Theorem 2, let $c_{1}$ and $c_{2}$ be positive integers, to be chosen later, and set

$$
\lambda_{i j}=\beta_{i j} \quad \text { for } 1 \leq i \leq 2 t \text { and } 1 \leq j \leq v_{i},
$$

while, for $1 \leq n \leq m$ and $1 \leq j \leq w_{n}$, set

$$
\mu_{n j}= \begin{cases}c_{1}^{j} \beta_{(2 t+1) j} & \text { for } n=1, \\ c_{2}^{j} \beta_{(2 t+2) j} & \text { for } n=2, \\ \beta_{(2 t+n) j} & \text { for } 3 \leq n \leq m .\end{cases}
$$

We then define, for $1 \leq i \leq 2 t$ and $1 \leq n \leq m$, the polynomials $f_{i}(x)$ and $g_{n}(y)$ by

$$
\begin{aligned}
& f_{i}(x)=\lambda_{i v_{i}} x^{v_{i}}+\lambda_{i\left(v_{i}-1\right)} x^{v_{i}-1}+\ldots+\lambda_{i 1} x \\
& g_{n}(y)=\mu_{n w_{n}} y^{w_{n}}+\mu_{n\left(w_{n}-1\right)} y^{w_{n}-1}+\ldots+\mu_{n 1} y .
\end{aligned}
$$

Note that the polynomials $f_{i}$ are of degree $v_{i}$ for $1 \leq i \leq 2 t$ and the polynomials $g_{n}$ are of degree $w_{n}$ for $1 \leq n \leq m$, that is, their leading coefficients are non-zero.

Now, in case (i) of Theorem 2, we choose large positive integers $c_{1}^{\prime}$ and $c_{2}^{\prime}$ so that

$$
1 \leq \frac{\left(c_{1}^{\prime}\right)^{w_{1}}}{\left(c_{2}^{\prime}\right)^{w_{2}}}\left|\frac{\beta_{(2 t+1) w_{1}}}{\beta_{(2 t+2) w_{2}}}\right| \leq 2
$$

to do so, first find a positive number $L$ that is large enough so that $(L+1)^{w_{1}} \leq 2 L^{w_{1}}$. Then choose $c_{2}^{\prime}$ to be a positive integer which is large enough so that

$$
\left(c_{2}^{\prime}\right)^{w_{2}}\left|\frac{\beta_{(2 t+2) w_{2}}}{\beta_{(2 t+1) w_{1}}}\right| \geq L^{w_{1}} .
$$

By our choice of $L$, we may choose a positive integer $c_{1}^{\prime}$ with $c_{1}^{\prime} \geq L$ for which (23) holds. Now set $c_{1}=u c_{1}^{\prime}$ and $c_{2}=u c_{2}^{\prime}$, where $u$ is a positive integer. Recalling the definitions (21) and (22), we may ensure that (14) holds by choosing $u$ sufficiently large. Note that by (22) and (23), we have $1 \leq\left|\mu_{1 w_{1}} / \mu_{2 w_{2}}\right| \leq 2$.

In case (ii) of Theorem 2, our choices are easier. We simply set $c_{2}=1$, and, recalling the definitions (21) and (22), we see that by choosing $c_{1}$ sufficiently large, we may ensure that (17) holds.

Note that in either case (i) or (ii), by (22), we have

$$
\frac{\mu_{1 j_{1}}}{\mu_{2 j_{2}}}=\frac{c_{1}^{j_{1}} \beta_{(2 t+1) j_{1}}}{c_{2}^{j_{2}} \beta_{(2 t+2) j_{2}}},
$$

which is irrational as $c_{1}$ and $c_{2}$ are positive integers. Also, recall that we have assumed that $\beta_{(2 t+1) d_{2 t+1}}>0$ and $\beta_{(2 t+2) d_{2 t+2}}<0$ in case (i) of Theorem 2 . 
In case (ii) of Theorem 2, we must have $\beta_{(2 t+1) d_{2 t+1}}>0$, as the polynomial $H(\mathbf{y})$ is positive-definite. It follows from the fact that $c_{1}$ and $c_{2}$ are positive integers, and the definition (22), that $\mu_{1 w_{1}}>0$ and $\mu_{2 w_{2}}<0$ in case (i) of Theorem 2, and that $\mu_{1 w_{1}}>0$ in case (ii) of Theorem 2 .

Now we apply cases (i) and (ii) of Proposition 1 to deduce, respectively, cases (i) and (ii) of Theorem 2. Note that our work above shows that the set of polynomials $f_{1}, \ldots, f_{2 t}, g_{1}, \ldots, g_{m}$ satisfy the respective assumptions of each case of the proposition. Thus, setting $P^{\prime}=P /\left(2 c_{1} c_{2}\right)$, and applying Proposition 1 for large $P^{\prime}$, we may see that for $|M| \leq \mu_{1 w_{1}} P^{\prime} / 8$ in case (i), and for $\mu_{1 w_{1}} P^{\prime} / 4 \leq M \leq 3 \mu_{1 w_{1}} P^{\prime} / 4$ in case (ii), we may find an integral solution $(\mathbf{x}, \mathbf{y})$ of the inequality

Then set

$$
\left|\sum_{i=1}^{2 t} f_{i}\left(x_{i}\right)+\sum_{n=1}^{m} g_{n}\left(y_{n}\right)-M\right|<\varepsilon .
$$

$$
z_{i}= \begin{cases}x_{i} & \text { for } 1 \leq i \leq 2 t \\ c_{1} y_{1} & \text { for } i=2 t+1 \\ c_{2} y_{2} & \text { for } i=2 t+2 \\ y_{i-2 t} & \text { for } 2 t+3 \leq i \leq 2 t+m \\ 0 & \text { for } i \geq 2 t+m+1\end{cases}
$$

(Observe that here we have again implicitly used the condition $s \geq s_{0}(k) \geq$ $2 t+m$.) By (21), (22) and (24), noting that $c_{1}$ and $c_{2}$ are integers, we find that $\mathbf{z}$ is an integral solution of $|H(\mathbf{z})-M|<\varepsilon$. Note also that if $(\mathbf{x}, \mathbf{y})$ is a non-trivial solution, then by $(24), \mathbf{z}$ is a non-trivial solution as well. Finally observe that because $|\mathbf{x}|,|\mathbf{y}| \leq 2 P^{\prime}$, the condition $|\mathbf{z}| \leq P$ follows from our choice of $P^{\prime}$.

This completes the proof that Proposition 1 implies Theorem 2. As we have remarked, Proposition 1 thus also implies Theorem 1.

3. The Davenport-Heilbronn method. We now start the proof of Proposition 1, which comprises the rest of the paper. We use the DavenportHeilbronn method, and in addition some of the recent ideas of Bentkus and Götze [2]. We also rely heavily on the methods of Davenport and Roth [10].

Before we begin, we need some standard notation. For real numbers $x$, we set

$$
e(x)=e^{2 \pi i x}
$$

At this point, we also take a moment to note that throughout the proof, all sums run only over integers. Also, implicit constants in the notations $\ll$, $\gg, o()$ and $O()$ may depend throughout on $k, t, \varepsilon$ and the coefficients $\lambda_{i j}$ and $\mu_{n j}$.

As is usual with the method, we need a special kernel function which allows us to give a lower bound for the number of solutions of the inequal- 
ity (13). We have the following lemma, essentially due to Davenport and Heilbronn. (See Lemma 4 of [8].)

Lemma 1 (Davenport and Heilbronn). Fix a positive number $\eta$. Then for any real number $\alpha$, define

$$
K(\alpha)=\frac{(\sin (\pi \eta \alpha))^{2}}{\pi^{2} \alpha^{2} \eta} .
$$

Observe that $K(\alpha)$ is a real-valued function which is positive and even. $K(\alpha)$ satisfies

$$
\int_{\mathbb{R}} e(\alpha t) K(\alpha) d \alpha=\max \left(0,1-\left|\frac{t}{\eta}\right|\right)
$$

for all real numbers $t$. Also, $K(\alpha)$ satisfies the bound

$$
K(\alpha) \ll_{\eta} \min \left(1,|\alpha|^{-2}\right) \text {. }
$$

Proof. The lemma can be deduced from the original result of Davenport and Heilbronn by a trivial change of variable.

For the remainder of the paper, we fix $K$ as in the lemma with the choice $\eta=\varepsilon$. Also, let $P$ be a large positive number; we shall require it to be sufficiently large at various points throughout the proof.

We now relabel the polynomials $f_{1}, \ldots, f_{2 t}$ to make our notation more convenient. Suppose that for each integer $j$ satisfying $2 \leq j \leq k$, there are $2 a_{j}$ polynomials of degree $j$, where $a_{j}$ is a non-negative integer. We assume, by relabeling, that the first $a_{k}$ polynomials $f_{1}, \ldots, f_{a_{k}}$ are of degree $k$, the next $a_{k-1}$ are of degree $k-1$ and so on, so that finally the polynomials $f_{t-a_{2}+1}, \ldots, f_{t}$ are of degree 2 . We relabel the polynomials $f_{t+1}, \ldots, f_{2 t}$ similarly, whence the degree of $f_{t+i}$ is the same as the degree of $f_{i}$ for $1 \leq i \leq t$. Observe that $a_{k}+a_{k-1}+\ldots+a_{2}=t$.

Now, for $2 \leq j \leq k$, define

$$
L_{j}=a_{k}+a_{k-1}+\ldots+a_{j+1} .
$$

Observe that the polynomials $f_{i}$ with $L_{j}+1 \leq i \leq L_{j}+a_{j}$ are the polynomials of degree $j$ among $f_{1}, \ldots, f_{t}$, if there are any at all, i.e., if $a_{j}$ is positive. Now we define exponents $\kappa_{i}$ for $1 \leq i \leq t$, as follows. For $i$ satisfying $L_{j}+1 \leq i \leq L_{j}+a_{j}$, we define

$$
\kappa_{i}=\kappa_{t+i}=\frac{1}{j-1}\left(\frac{j-1}{j}\right)^{i-L_{j}} \prod_{l=j+1}^{k}\left(\frac{l-1}{l}\right)^{a_{l}} .
$$

Observe that we of course only define such exponents if $a_{j}$ is positive, and the product above is of course understood to be one in the case when $j=k$. Essentially, these are the natural exponents to be used in a diminishing ranges argument involving polynomials of different degrees. Also, for 
$1 \leq i \leq t$, define

$$
P_{i}=P^{\kappa_{i}}
$$

Now we collect some observations about the exponents $\kappa_{i}$ and the numbers $P_{i}$ which will be important for future reference. Note from (27) that for $L_{j}+1 \leq i \leq L_{j}+a_{j}$, recalling that $v_{i}$ is the degree of the polynomial $f_{i}$, we have

$$
\kappa_{i} v_{i} \leq \frac{j}{j-1}\left(\frac{j-1}{j}\right)^{i-L_{j}} \leq 1 .
$$

It follows that

$$
P_{i}^{v_{i}} \leq P \quad \text { for } 1 \leq i \leq t .
$$

Observe that for $L_{j}+1 \leq i \leq L_{j}+a_{j}-1$, we have $\kappa_{i+1}=\kappa_{i}((j-1) / j)$. Note that in this case, $v_{i+1}=v_{i}=j$, so $\kappa_{i+1} v_{i+1}=\kappa_{i}\left(v_{i}-1\right)$. For $i=L_{j}+a_{j}$, assuming that $1 \leq i \leq t-1$ and that $r$ is the largest integer less than $j$ for which $a_{r}>0$, we have $\kappa_{i+1}=\kappa_{i}((j-1) / r)$. In this case, $v_{i}=j$, whereas $v_{i+1}=r$, so $\kappa_{i+1} v_{i+1}=\kappa_{i}\left(v_{i}-1\right)$ again. Thus

$$
\kappa_{i+1} v_{i+1}=\kappa_{i}\left(v_{i}-1\right) \quad \text { for } 1 \leq i \leq t-1 .
$$

By the definition (28) of $P_{i}$, we therefore have

$$
P_{i+1}^{v_{i+1}}=P_{i}^{v_{i}-1} \quad \text { for } 1 \leq i \leq t-1 .
$$

Finally, we consider the sum $\sum_{i=1}^{t} \kappa_{i}$. One obtains

$$
\begin{aligned}
\sum_{i=1}^{t} \kappa_{i} & =\sum_{j=2}^{k} \frac{1}{j-1}\left(\prod_{l=j+1}^{k}\left(\frac{l-1}{l}\right)^{a_{l}}\right) \sum_{i=1}^{a_{j}}\left(\frac{j-1}{j}\right)^{i} \\
& =\sum_{j=2}^{k} \frac{1}{j-1}\left(\prod_{l=j+1}^{k}\left(\frac{l-1}{l}\right)^{a_{l}}\right)(j-1)\left(1-\left(\frac{j-1}{j}\right)^{a_{j}}\right) \\
& =\sum_{j=2}^{k}\left(\prod_{l=j+1}^{k}\left(\frac{l-1}{l}\right)^{a_{l}}-\prod_{l=j}^{k}\left(\frac{l-1}{l}\right)^{a_{l}}\right) \\
& =\left(1-\prod_{l=2}^{k}\left(\frac{l-1}{l}\right)^{a_{l}}\right) .
\end{aligned}
$$

From this calculation, we clearly have

$$
\sum_{i=1}^{t} \kappa_{i}=\left(1-\prod_{l=2}^{k}\left(\frac{l-1}{l}\right)^{a_{l}}\right) \geq\left(1-\left(\frac{k-1}{k}\right)^{t}\right) .
$$

Therefore from (12) one obtains

$$
\sum_{i=1}^{t} \kappa_{i}>1-\frac{(2 k+1) \phi}{k}
$$


Now we define the generating functions we shall use. For $1 \leq i \leq t$ and any real number $\alpha$, we define

$$
\begin{gathered}
S_{i}(\alpha)=S_{i}(\alpha, P)=\sum_{P_{i} \leq x \leq 2 P_{i}} e\left(\alpha f_{i}(x)\right), \\
S_{t+i}(\alpha)=S_{t+i}(\alpha, P)=\sum_{P_{i} \leq x \leq 2 P_{i}} e\left(\alpha f_{t+i}(x)\right) .
\end{gathered}
$$

Also, for any real numbers $\alpha$ and $N$, and any polynomial $g(x)$, we set

$$
U(\alpha, g)=U(\alpha, g, N)=\sum_{1 \leq x \leq N} e(\alpha g(x)) .
$$

Recall that $w_{n}$ is the degree of the polynomial $g_{n}$. For $1 \leq n \leq m$ and any real number $\alpha$, we define

$$
U_{n}(\alpha)=U_{n}(\alpha, P)=U\left(\alpha, g_{n}, P^{1 / w_{n}}\right) .
$$

Also define

$$
W=\sum_{n=1}^{m} \frac{1}{w_{n}} .
$$

Note that $1 / w_{n} \geq 1 / k$ and $m=2 k+4$, whence clearly

$$
W \geq(2 k+4) / k \text {. }
$$

Considering all of the above definitions and the identity (25), one may see in a standard manner that the number of integral solutions $(\mathbf{x}, \mathbf{y})$ of the inequality (13) with $|\mathbf{x}| \leq 2 P$ and $|\mathbf{y}| \leq 2 P$ is at least

$$
\int_{\mathbb{R}}\left(\prod_{i=1}^{2 t} S_{i}(\alpha)\right)\left(\prod_{n=1}^{m} U_{n}(\alpha)\right) e(-M \alpha) K(\alpha) d \alpha .
$$

We will show that in fact this integral is $\gg\left(P_{1} \ldots P_{t}\right)^{2} P^{W-1}$ for large $P$, which in turn certainly shows that there is a non-trivial solution of the inequality (13). So for all large $P$, we will in fact have an asymptotic lower bound of this type for the number of solutions of the inequality (13); this is one of the benefits of using the techniques of Bentkus and Götze. With the standard Davenport-Heilbronn method, we would only obtain such an asymptotic lower bound for a sequence of large $P$ tending to infinity.

As is usual, our strategy will be to give a dissection of the real line into three regions, and consider the contributions to the integral (37) from each of the three regions. For the remainder of the paper, we fix a positive number $\delta$ satisfying

$$
0<\delta<1 / k
$$

We could fix a value of $\delta$ for definiteness if we so desired, but we choose not 
to. We then define the region $\mathcal{M}$, which we call the major arc, by setting

$$
\mathcal{M}=\left\{\alpha \in \mathbb{R}:|\alpha| \leq P^{\delta-1}\right\} .
$$

In Section 7, we will show that the contribution to the integral (37) from the major arc is $\gg\left(P_{1} \ldots P_{t}\right)^{2} P^{W-1}$ for large $P$. During the course of the proof, we shall define a function $T(P)$ which depends only on the coefficients $\mu_{n j}$, and tends to infinity as $P$ tends to infinity. Using this function, we define the so-called minor arcs $\mathfrak{m}$ by

$$
\mathfrak{m}=\left\{\alpha \in \mathbb{R}: P^{\delta-1}<|\alpha| \leq T(P)\right\} .
$$

This will be the most difficult region to treat. Finally, we define the so-called trivial arcs $\mathfrak{t}$ by

$$
\mathfrak{t}=\{\alpha \in \mathbb{R}:|\alpha|>T(P)\} .
$$

We will show in Section 6 that the contribution to the integral (37) from each of the last two regions is $o\left(\left(P_{1} \ldots P_{t}\right)^{2} P^{W-1}\right)$. Combined with our treatment of the major arc, this will show that the integral $(37)$ is $\gg\left(P_{1} \ldots P_{t}\right)^{2} P^{W-1}$ for large $P$. We now proceed to the treatment of the minor arcs and trivial arcs, by establishing an analogue of Hua's inequality.

4. An analogue of Hua's inequality. We first give a version of a lemma essentially proved originally by Vinogradov. We state the lemma in a form very close to that given by Baker. (See Theorem 4.4 of [1].)

Lemma 2. Fix a positive number $\eta$. Suppose that $k$ is an integer satisfying $k \geq 2$. Let $J(k)=8 k^{2}(\log k+(1 / 2) \log \log k+2)$. Suppose that $N$ is a positive number which is sufficiently large in terms of $k$. For real numbers $\alpha_{1}, \ldots, \alpha_{k}$, let $f(x)=\alpha_{k} x^{k}+\alpha_{k-1} x^{k-1}+\ldots+\alpha_{1} x$. Suppose also that

$$
\left|\sum_{1 \leq x \leq N} e(f(x))\right| \geq \gamma N,
$$

where $\gamma$ is a positive number satisfying

$$
\gamma \geq N^{-1 / J(k)}
$$

Then there are integers $y, u_{1}, \ldots, u_{k}$ with

$$
1 \leq y \leq \gamma^{-k} N^{\eta} \quad \text { and } \quad\left|y \alpha_{j}-u_{j}\right| \leq \gamma^{-k} N^{\eta-j} \quad \text { for } 1 \leq j \leq k,
$$

and

$$
\left(y, u_{k}, u_{k-1}, \ldots, u_{2}\right) \leq 2 k^{2}, \quad\left(y, u_{k}, u_{k-1}, \ldots, u_{1}\right)=1 .
$$

The lemma can be obtained by adjustments of the proof of the case $M=1$ of Theorem 4.4 of [1]; we give a proof for the sake of completeness, although we will be brief. We also note that the bounds of the lemma could be improved by combining the proof with the work of Wooley [22], but a superior version of this type does not yield significant improvement of our final results. 
Proof of Lemma 2. We first consider the case $k \geq 4$. As in the proof of Theorem 4.4 of [1], we choose $l=\left[k \log \left(4 k^{2} \log k\right)\right]+1$. One has $J(k)>$ $4 k l(1-2 \theta)^{-1}$ exactly as in that proof, where

$$
\theta=\theta(k)=\frac{1}{2}(k-1)^{2}\left(\frac{k-2}{k-1}\right)^{l} .
$$

Set $A=\gamma N$. Then it follows from (42) that

$$
\left(N A^{-1}\right)^{4(k-1) l} \ll N^{4(k-1) l / J(k)} \ll N^{1-2 \theta-4 \eta}
$$

for sufficiently small $\eta$.

Now consider the cases $k=2$ and $k=3$. We set $l=3$ in these cases, whence $\theta(2)=0$ and $\theta(3)=1 / 4$, where $\theta(k)$ is defined as above. We set $A=\gamma N$. A calculation reveals that $J(2) \geq 80$ and $J(3) \geq 226$. Thus, from (42), one can check that the condition (43) also holds in these cases.

Now we consider all of the cases $k \geq 2$ simultaneously. As (43) holds for $k \geq 2$, we can apply Theorem 4.3 of [1] with $A=\gamma N$ for large $N$. It follows that there are coprime pairs of integers $q_{j}$ and $a_{j}$ for $2 \leq j \leq k$ such that

$$
q_{j} \geq 1 \quad \text { and } \quad\left|q_{j} \alpha_{j}-a_{j}\right| \leq N^{\eta-j+\theta}\left(N A^{-1}\right)^{2(k-1) l} \quad \text { for } 2 \leq j \leq k,
$$

and such that the least common multiple $q_{0}$ of $q_{2}, \ldots, q_{k}$ satisfies

$$
q_{0} \leq\left(N A^{-1}\right)^{2(k-1) l} N^{\theta+\eta} .
$$

As the condition (43) holds for $k \geq 2$, it follows that for sufficiently small $\eta$ we have

$$
1 \leq q_{0} \leq N^{1-2 \eta}
$$

and that there are integers $b_{j}$ for $2 \leq j \leq k$ such that

$$
\left|q_{0} \alpha_{j}-b_{j}\right| \ll N^{1-j-2 \eta} \quad \text { for } 2 \leq j \leq k ;
$$

in fact, one simply chooses $b_{j}=q_{0} a_{j} / q_{j}$ for $2 \leq j \leq k$. We note that by a simple argument one also has

$$
\left(q_{0}, b_{k}, \ldots, b_{2}\right)=1 \text {. }
$$

Now, following the proof of Theorem 4.4 of [1], for large $N$ we may apply Lemma 4.6 of [1] with $r=q_{0}$ and $H=A$, noting that $d=1$. We then set $y=t q_{0}$, and $u_{j}=t b_{j}$ for $2 \leq j \leq k$, and let $u_{1}$ be the integer closest to $t q_{0} \alpha_{1}$. If necessary, we can remove a factor so that we also have $\left(y, u_{k}, u_{k-1}, \ldots, u_{1}\right)=1$. This completes the proof of Lemma 2 .

We now need a generalization of a lemma due to Davenport and Roth. (See Lemma 3 of [10].) We give a proof for completeness, although our proof is similar to theirs. 
Lemma 3. Suppose that we are in the setting of Proposition 1, and that $S_{i}(\alpha)$ is defined as in (32). Then

$$
\int_{\mathbb{R}}\left(\prod_{i=1}^{2 t}\left|S_{i}(\alpha)\right|\right) K(\alpha) d \alpha \ll P_{1} \ldots P_{t} .
$$

Proof. We first use the Cauchy-Schwarz inequality, bearing in mind that $K$ is positive, whence we see that the left hand side of (44) is

$$
\ll\left(\int_{\mathbb{R}}\left(\prod_{i=1}^{t}\left|S_{i}(\alpha)\right|^{2}\right) K(\alpha) d \alpha\right)^{1 / 2}\left(\int_{\mathbb{R}}\left(\prod_{i=t+1}^{2 t}\left|S_{i}(\alpha)\right|^{2}\right) K(\alpha) d \alpha\right)^{1 / 2} .
$$

We shall show that

$$
\int_{\mathbb{R}}\left(\prod_{i=1}^{t}\left|S_{i}(\alpha)\right|^{2}\right) K(\alpha) d \alpha \ll P_{1} \ldots P_{t}
$$

A similar bound holds for the other integral in (45). We omit its proof as the method is the same.

Using the identity (25) and the definition (32), one can see that the integral in (46) is less than or equal to the number of integral solutions $(\mathbf{x}, \mathbf{y})=\left(x_{1}, \ldots, x_{t}, y_{1}, \ldots, y_{t}\right)$ of the inequality

$$
\left|f_{1}\left(x_{1}\right)-f_{1}\left(y_{1}\right)+f_{2}\left(x_{2}\right)-f_{2}\left(y_{2}\right)+\ldots+f_{t}\left(x_{t}\right)-f_{t}\left(y_{t}\right)\right|<\varepsilon,
$$

where one has $P_{i} \leq x_{i}, y_{i} \leq 2 P_{i}$ for $1 \leq i \leq t$. We bound the number of such solutions by induction.

First, we make a general observation. If $x$ and $y$ are positive numbers with $x>y$ and $l$ is a positive integer, then

$$
x^{l}-y^{l} \geq l(x-y) y^{l-1} .
$$

Also, recall from (30) that $P_{i+1}^{v_{i+1}}=P_{i}^{v_{i}-1}$ for $1 \leq i \leq t-1$. It follows by induction that given an integer $i_{0}$ with $1 \leq i_{0} \leq t-1$, we have

$$
P_{i}^{v_{i}} \leq P_{i_{0}}^{v_{i_{0}}-1} \quad \text { for } i_{0}<i \leq t
$$

Now fix any choice of $y_{1}$ which satisfies $P_{1} \leq y_{1} \leq 2 P_{1}$. Suppose that there are integers $x_{1}, x_{2}, \ldots, x_{t}, y_{2}, \ldots, y_{t}$, with $P_{i} \leq x_{i}, y_{i} \leq 2 P_{i}$ for $2 \leq i \leq t$ and $P_{1} \leq x_{1} \leq 2 P_{1}$, such that $(\mathbf{x}, \mathbf{y})$ is a solution of (47). By (49), one has

$$
\left|\sum_{i=2}^{t}\left(f_{i}\left(x_{i}\right)-f_{i}\left(y_{i}\right)\right)\right| \ll P_{1}^{v_{1}-1} ;
$$

here the implied constant in Vinogradov's notation may depend on the coefficients $\lambda_{i j}$, but not on $P$. Now we also have $\left|f_{1}\left(x_{1}\right)-\lambda_{1 v_{1}} x_{1}^{v_{1}}\right| \ll P_{1}^{v_{1}-1}$ and $\left|f_{1}\left(y_{1}\right)-\lambda_{1 v_{1}} y_{1}^{v_{1}}\right| \ll P_{1}^{v_{1}-1}$, whence

$$
\left|\lambda_{1 v_{1}}\left(x_{1}^{v_{1}}-y_{1}^{v_{1}}\right)\right| \ll P_{1}^{v_{1}-1} .
$$

As $y_{1} \geq P_{1}$ and $x_{1} \geq P_{1}$ and $\lambda_{1 v_{1}}$ is non-zero, by the observation (48) there are only finitely many possible choices $x_{1}$, given this fixed choice 
of $y_{1}$, for which the vector $\left(x_{1}, \ldots, x_{t}, y_{1}, \ldots, y_{t}\right)$ could possibly be a solution of the inequality (47). So there are $\ll P_{1}$ choices of the integral vector $\left(x_{1}, y_{1}\right)$, with $P_{1} \leq x_{1}, y_{1} \leq 2 P_{1}$, which could extend to an integral solution $\left(x_{1}, \ldots, x_{t}, y_{1}, \ldots, y_{t}\right)$ of $(47)$, with $P_{i} \leq x_{i}, y_{i} \leq 2 P_{i}$ for $1 \leq i \leq t$.

Now assume that for some $j$ with $2 \leq j \leq t$, there are $\ll P_{1} \ldots P_{j-1}$ choices of the integral vector $\left(x_{1}, \ldots, x_{j-1}, y_{1}, \ldots, y_{j-1}\right)$, with $P_{i} \leq x_{i}, y_{i}$ $\leq 2 P_{i}$ for $1 \leq i \leq j-1$, which could extend to an integral solution $\left(x_{1}, \ldots, x_{j-1}, \ldots, x_{t}, y_{1}, \ldots, y_{j-1}, \ldots, y_{t}\right)$ of $(47)$, with $P_{i} \leq x_{i}, y_{i} \leq 2 P_{i}$ for $1 \leq i \leq t$. Note this does indeed hold in the case $j=2$, as we have demonstrated. We aim of course to establish this result for the case $j+1$.

To this end, fix a choice $\left(x_{1}, \ldots, x_{j-1}, y_{1}, \ldots, y_{j-1}\right)$ as above. Also fix a choice of $y_{j}$ with $P_{j} \leq y_{j} \leq 2 P_{j}$. Then suppose that the vector given by $\left(x_{1}, \ldots, x_{j}, \ldots, x_{t}, y_{1}, \ldots, y_{j}, \ldots, y_{t}\right)$ is a solution of the inequality (47) with $P_{i} \leq x_{i}, y_{i} \leq 2 P_{i}$ for $j+1 \leq i \leq t$ and with $P_{j} \leq x_{j} \leq 2 P_{j}$. Then, as above,

$$
\left|\lambda_{j v_{j}}\left(x_{j}^{v_{j}}-y_{j}^{v_{j}}\right)+\sum_{i=1}^{j-1}\left(f_{i}\left(x_{i}\right)-f_{i}\left(y_{i}\right)\right)\right| \ll P_{j}^{v_{j}-1} .
$$

By (48), the integers $z^{v_{j}}$ with $P_{j} \leq z \leq 2 P_{j}$ are spaced apart by at least some constant multiple of $P_{j}^{v_{j}-1}$, whence, because $\lambda_{j v_{j}}$ is non-zero, for a fixed choice of $y_{j}$, there are finitely many choices of $x_{j}$ for which the last bound could possibly hold. Thus there are $\ll P_{1} \ldots P_{j}$ possible choices of the (2j)-tuple $\left(x_{1}, \ldots, x_{j}, y_{1}, \ldots, y_{j}\right)$ which could possibly extend to a solution $(\mathbf{x}, \mathbf{y}) \in \mathbb{Z}^{2 t}$ of the inequality (47) with the constraint $P_{i} \leq x_{i}, y_{i} \leq 2 P_{i}$ on the variables.

Therefore by induction we obtain the desired bound (46) and hence complete the proof of Lemma 3 .

We now quote a lemma. (See Lemma 4.4 of [1].)

Lemma 4 (Baker). Fix a positive number $\eta$. Suppose that $k$ is an integer with $k \geq 2$ and that $N$ is a real number with $N \geq 1$. For real numbers $\alpha_{1}, \ldots, \alpha_{k}$, let $f(x)=\alpha_{k} x^{k}+\ldots+\alpha_{1} x$ and suppose that $q, a_{1}, \ldots, a_{k}$ are integers with

$$
\left|q \alpha_{j}-a_{j}\right| \leq \frac{N^{1-j}}{2 k^{2}} \quad \text { for } 1 \leq j \leq k .
$$

Set $d=\left(q, a_{k}, \ldots, a_{2}\right)$ and write

$$
\begin{gathered}
\beta_{j}=\alpha_{j}-\frac{a_{j}}{q} \quad \text { for } 1 \leq j \leq k, \quad g(x)=\sum_{j=1}^{k} \beta_{j} x^{j}, \\
G(x)=\sum_{j=1}^{k} a_{j} x^{j}, \quad S(q)=\sum_{v=1}^{q} e\left(\frac{G(v)}{q}\right) .
\end{gathered}
$$


Then

$$
\sum_{x=1}^{T} e(f(x))=\frac{S(q)}{q} \int_{0}^{T} e(g(y)) d y+O\left(q^{1-1 / k+\eta} d^{1 / k}\right)
$$

for all real numbers $T$ with $1 \leq T \leq N$.

Note that Baker only states the above lemma for integers $T$, but the result clearly extends to real numbers $T$ by absorbing a number of size at most 1 on each side into the error term.

We can now give the central lemma of this section. It is our version of Hua's inequality.

Lemma 5. Suppose that we are in the setting of Proposition 1. Define the functions $S_{i}(\alpha)$ as in (32) and the functions $U(\alpha, g)$ as in (33). Also let $K(\alpha)$ be as in Lemma 1 with $\eta=\varepsilon$. Suppose that $r$ is an integer satisfying $r \geq 2 k+1$. Then for $1 \leq n \leq r$, let $w_{n}$ be an integer satisfying $2 \leq w_{n} \leq k$ and let

$$
h_{n}(x)=\gamma_{n w_{n}} x^{w_{n}}+\gamma_{n\left(w_{n}-1\right)} x^{w_{n}-1}+\ldots+\gamma_{n 1} x
$$

be a real polynomial with zero constant term, where $\gamma_{n w_{n}} \neq 0$ for $1 \leq n \leq r$. Let

Then

$$
V=\sum_{n=1}^{r} \frac{1}{w_{n}}
$$

$$
\int_{\mathbb{R}}\left(\prod_{i=1}^{2 t}\left|S_{i}(\alpha)\right|\right)\left(\prod_{n=1}^{r}\left|U\left(\alpha, h_{n}, P^{1 / w_{n}}\right)\right|\right) K(\alpha) d \alpha \ll\left(P_{1} \ldots P_{t}\right)^{2} P^{V-1} .
$$

We note that for many applications of such an inequality, a weaker bound of the type $\left(P_{1} \ldots P_{t}\right)^{2} P^{V-1+\eta}$, where this holds for any small positive number $\eta$, would be sufficient. Such a bound does not suffice for our purposes however, since we are using some of the techniques of Bentkus and Götze.

Proof of Lemma 5. We essentially use the Hardy-Littlewood method, in a way which is not exactly standard. Thus we shall give the whole proof.

Observe that

$$
\sum_{n=1}^{r} \frac{1}{V w_{n}}=1
$$

Thus by Hölder's inequality, and the fact that $K(\alpha)$ is positive, we may see that the integral in (51) satisfies

$$
\begin{aligned}
\int_{\mathbb{R}}\left(\prod_{i=1}^{2 t}\left|S_{i}(\alpha)\right|\right)\left(\prod_{n=1}^{r}\left|U\left(\alpha, h_{n}, P^{1 / w_{n}}\right)\right|\right) K(\alpha) d \alpha \\
\quad \ll \prod_{n=1}^{r}\left(\int_{\mathbb{R}}\left(\prod_{i=1}^{2 t}\left|S_{i}(\alpha)\right|\right)\left|U\left(\alpha, h_{n}, P^{1 / w_{n}}\right)\right|^{V w_{n}} K(\alpha) d \alpha\right)^{1 /\left(V w_{n}\right)} .
\end{aligned}
$$


Therefore, in order to prove the lemma, it suffices to show that

$$
\int_{\mathbb{R}}\left(\prod_{i=1}^{2 t}\left|S_{i}(\alpha)\right|\right)\left|U\left(\alpha, h_{n}, P^{1 / w_{n}}\right)\right|^{V w_{n}} K(\alpha) d \alpha \ll\left(P_{1} \ldots P_{t}\right)^{2} P^{V-1}
$$

for $1 \leq n \leq r$.

We thus fix $n$ with $1 \leq n \leq r$ and prove (52). Fix as well a positive number $\eta$, which we shall later choose to be sufficiently small. For convenience of notation throughout the proof, we set

$$
u=w_{n} .
$$

Recall the definition (11) of $\phi$. We now give some other definitions. For integers $q$ and $a_{1}, \ldots, a_{u}$ with $q \geq 1$, we write $\mathbf{a}=\left(a_{1}, \ldots, a_{u}\right)$ and define

$$
\mathcal{N}(q, \mathbf{a})=\left\{\alpha \in \mathbb{R}:\left|\gamma_{n j} \alpha q-a_{j}\right|<P^{\phi-j / u} \text { for } 1 \leq j \leq u\right\} .
$$

For any integers $q$ and $a_{u}$ with $q \geq 1$, we define

$$
\mathcal{N}_{0}\left(q, a_{u}\right)=\left\{\alpha \in \mathbb{R}:\left|\gamma_{n u} \alpha q-a_{u}\right|<P^{\phi-1}\right\}
$$

Now, set

$$
\mathcal{N}=\bigcup_{1 \leq q \leq P^{\phi}} \bigcup_{\substack{\text { a: }\left(q, a_{u}, \ldots, a_{1}\right)=1 \\\left(q, a_{u}, \ldots, a_{2}\right) \leq 2 u^{2}}} \mathcal{N}(q, \mathbf{a}) .
$$

For the purposes of this lemma, we think of $\mathcal{N}$ as a version of the major arcs. Now we define a corresponding version of the minor arcs, namely the set

$$
\mathfrak{n}=\mathbb{R} \backslash \mathcal{N} .
$$

We show first that

$$
\int_{\mathfrak{n}}\left(\prod_{i=1}^{2 t}\left|S_{i}(\alpha)\right|\right)\left|U\left(\alpha, h_{n}, P^{1 / u}\right)\right|^{u V} K(\alpha) d \alpha \ll\left(P_{1} \ldots P_{t}\right)^{2} P^{V-1} .
$$

In fact, we show that the left side is strictly smaller in order than the right side, although this is not needed.

Suppose now that $\alpha \in \mathfrak{n}$, and that $\left|U\left(\alpha, h_{n}, P^{1 / u}\right)\right| \geq P^{1 / u-\phi / u+\eta}$. We can then apply Lemma 2 with $N=P^{1 / u}$, with $\gamma=P^{-\phi / u+\eta}$ and $k=u$, and with $\alpha_{j}=\alpha \gamma_{n j}$. Note that we have assumed $u \geq 2$. We obtain integers $q, a_{1}, \ldots, a_{u}$ with

$$
1 \leq q \leq P^{\phi}, \quad\left|\gamma_{n j} \alpha q-a_{j}\right|<P^{\phi-j / u} \quad \text { for } 1 \leq j \leq u,
$$

and

$$
\left(q, a_{u}, \ldots, a_{2}\right) \leq 2 u^{2}, \quad\left(q, a_{u}, \ldots, a_{1}\right)=1 .
$$

But then $\alpha \in \mathcal{N}$, which contradicts the assumption $\alpha \in \mathfrak{n}$. Therefore

$$
\left|U\left(\alpha, h_{n}, P^{1 / u}\right)\right| \leq P^{1 / u-\phi / u+\eta} \quad \text { for } \alpha \in \mathfrak{n} .
$$


It follows from Lemma 3 that

$$
\begin{aligned}
\int_{\mathfrak{n}}\left(\prod_{i=1}^{2 t}\left|S_{i}(\alpha)\right|\right) \mid & \left.U\left(\alpha, h_{n}, P^{1 / u}\right)\right|^{u V} K(\alpha) d \alpha \\
& \ll \sup _{\alpha \in \mathfrak{n}}\left|U\left(\alpha, h_{n}, P^{1 / u}\right)\right|^{u V} \int_{\mathfrak{n}}\left(\prod_{i=1}^{2 t}\left|S_{i}(\alpha)\right|\right) K(\alpha) d \alpha \\
& \ll P^{V-V \phi+u V \eta} P_{1} \ldots P_{t} .
\end{aligned}
$$

Now, by (28), we have

$$
P^{V-V \phi+u V \eta} P_{1} \ldots P_{t}=P^{\Gamma}\left(P_{1} \ldots P_{t}\right)^{2},
$$

where

$$
\Gamma=V-V \phi+u V \eta-\sum_{i=1}^{t} \kappa_{i}
$$

By (31), for sufficiently small $\eta$ we have

$$
\Gamma<V-V \phi-1+(2 k+1) \phi / k .
$$

Observe that

$$
V=\sum_{n=1}^{r} \frac{1}{w_{n}} \geq \sum_{n=1}^{r} \frac{1}{k} \geq \frac{2 k+1}{k}
$$

Thus

$$
\Gamma<V-1 \text {. }
$$

By (54) and (55), the bound (53) follows.

To obtain the bound (52) and thus to finish the proof of the lemma, it remains only to prove that

$$
\int_{\mathcal{N}}\left(\prod_{i=1}^{2 t}\left|S_{i}(\alpha)\right|\right)\left|U\left(\alpha, h_{n}, P^{1 / u}\right)\right|^{u V} K(\alpha) d \alpha \ll\left(P_{1} \ldots P_{t}\right)^{2} P^{V-1} .
$$

We make a few observations which enable us to prove this bound.

First, suppose that $\alpha \in \mathcal{N}(q, \mathbf{a}) \subseteq \mathcal{N}$. We may see from (11) that $u \phi \leq$ $k \phi<1$. Moreover, $P$ is large, so we may apply Lemma 4 with $k=u$ and $N=P^{1 / u}$, and thus we obtain

$$
U\left(\alpha, h_{n}, P^{1 / u}\right)=\frac{S(q)}{q} \int_{0}^{P^{1 / u}} e(g(y)) d y+O\left(q^{1-1 / u+\eta}\right)
$$

where

$$
\begin{gathered}
\beta_{j}=\gamma_{n j} \alpha-\frac{a_{j}}{q} \quad \text { for } 1 \leq j \leq u, \quad g(y)=\sum_{j=1}^{u} \beta_{j} y^{j} \\
G(x)=\sum_{j=1}^{u} a_{j} x^{j}, \quad S(q)=\sum_{v=1}^{q} e\left(\frac{G(v)}{q}\right) .
\end{gathered}
$$


By Theorems 7.1 and 7.3 of [18], for $\alpha \in \mathcal{N}(q, \mathbf{a}) \subseteq \mathcal{N}$, we therefore have $U\left(\alpha, h_{n}, P^{1 / u}\right)$
$\quad \ll q^{-1 / u+\eta} P^{1 / u}\left(1+\left|\beta_{1}\right| P^{1 / u}+\left|\beta_{2}\right| P^{2 / u}+\ldots+\left|\beta_{u}\right| P\right)^{-1 / u}+q^{1-1 / u+\eta}$.

It certainly follows that

$$
U\left(\alpha, h_{n}, P^{1 / u}\right) \ll q^{-1 / u+\eta}\left(\min \left(P^{1 / u},\left|\beta_{u}\right|^{-1 / u}\right)+q\right),
$$

whence also

$$
U\left(\alpha, h_{n}, P^{1 / u}\right)^{u V} \ll q^{-V+u V \eta}\left(\left(\min \left(P^{1 / u},\left|\beta_{u}\right|^{-1 / u}\right)\right)^{u V}+q^{u V}\right) .
$$

For any integers $q$ and $a_{u}$ with $q \geq 1$, define

$$
\mathcal{R}\left(q, a_{u}\right)=\bigcup \mathcal{N}(q, \mathbf{a}),
$$

where the union is taken over all integer $(u-1)$-tuples $\left(a_{1}, a_{2}, \ldots, a_{u-1}\right)$ such that

$$
\left(q, a_{u}, \ldots, a_{1}\right)=1 \quad \text { and }\left(q, a_{u}, \ldots, a_{2}\right) \leq 2 u^{2} .
$$

Then we certainly have

$$
\mathcal{R}\left(q, a_{u}\right) \subset \mathcal{N}_{0}\left(q, a_{u}\right)
$$

We need to make one more observation. Suppose that $\alpha \in \mathcal{N}(q, \mathbf{a}) \subseteq \mathcal{N}$. Then $\left|\gamma_{n u} \alpha q-a_{u}\right|<P^{\phi-1}$. But $\gamma_{n u}$ is non-zero, as the polynomial $h_{n}$ has degree $u$. One certainly has $\phi<1$, whence for non-zero $a_{u}$ and sufficiently large $P$ we have

$$
|\alpha| \geq \frac{\left|a_{u}\right|}{2 q\left|\gamma_{n u}\right|} \gg \frac{\left|a_{u}\right|}{q} .
$$

Note on the other hand that this statement trivially follows if $a_{u}=0$. So it holds regardless of the value of $a_{u}$.

We now consider the integral in (57). By the definitions of $\mathcal{N}$ and $\mathcal{R}\left(q, a_{u}\right)$, by trivial bounds, and by noting that $K$ is positive, we have

$$
\begin{aligned}
\int_{\mathcal{N}}\left(\prod_{i=1}^{2 t}\left|S_{i}(\alpha)\right|\right)\left|U\left(\alpha, h_{n}, P^{1 / u}\right)\right|^{u V} K(\alpha) d \alpha & \\
& \ll\left(P_{1} \ldots P_{t}\right)^{2} \sum_{1 \leq q \leq P^{\phi}} \sum_{a_{u} \in \mathbb{Z} \mathcal{R}\left(q, a_{u}\right)} \int_{\mid}\left|U\left(\alpha, h_{n}, P^{1 / u}\right)\right|^{u V} K(\alpha) d \alpha .
\end{aligned}
$$

Now for $\alpha \in \mathcal{R}\left(q, a_{u}\right)$, one has $\alpha \in \mathcal{N}(q, \mathbf{a})$ for some integers $a_{1}, \ldots, a_{u-1}$ which satisfy the conditions $\left(q, a_{u}, \ldots, a_{1}\right)=1$ and $\left(q, a_{u}, \ldots, a_{2}\right) \leq 2 u^{2}$. Thus (60) holds, whence by the bound (26) we have

$$
\begin{aligned}
& \int_{\mathcal{N}}\left(\prod_{i=1}^{2 t}\left|S_{i}(\alpha)\right|\right)\left|U\left(\alpha, h_{n}, P^{1 / u}\right)\right|^{u V} K(\alpha) d \alpha \\
& \ll\left(P_{1} \ldots P_{t}\right)^{2} \sum_{1 \leq q \leq P^{\phi}} \sum_{a_{u} \in \mathbb{Z}} \min \left(1,\left|a_{u}\right|^{-2} q^{2}\right) \int_{\mathcal{R}\left(q, a_{u}\right)}\left|U\left(\alpha, h_{n}, P^{1 / u}\right)\right|^{u V} d \alpha .
\end{aligned}
$$


Now for $\alpha \in \mathcal{R}\left(q, a_{u}\right)$ and $q \leq P^{\phi}$, we have $\alpha \in \mathcal{N}(q, \mathbf{a})$ for some integers $a_{1}, \ldots, a_{u-1}$ satisfying the conditions $\left(q, a_{u}, \ldots, a_{1}\right)=1$ and $\left(q, a_{u}, \ldots, a_{2}\right) \leq$ $2 u^{2}$, so the bound (58) holds. Using also (59) and the fact that $q \leq P^{\phi}$, we obtain

$$
\begin{aligned}
\int_{\mathcal{R}\left(q, a_{u}\right)}\left|U\left(\alpha, h_{n}, P^{1 / u}\right)\right|^{u V} d \alpha & \ll \int_{\mathcal{N}_{0}\left(q, a_{u}\right)} q^{-V+u V \eta}\left(\left(\min \left(P^{1 / u},\left|\beta_{u}\right|^{-1 / u}\right)\right)^{u V}+q^{u V}\right) d \alpha \\
& \ll \int_{0}^{q^{-1} P^{\phi-1}} q^{-V+u V \eta}\left(\left(\min \left(P^{1 / u}, \beta_{u}^{-1 / u}\right)\right)^{u V}+q^{u V}\right) d \beta_{u} \\
& \ll q^{-V+u V \eta}\left[\int_{0}^{P^{-1}} P^{V} d \beta_{u}+\int_{P^{-1}}^{\infty} \beta_{u}^{-V} d \beta_{u}+q^{u V-1} P^{\phi-1}\right] \\
& \ll q^{-V+u V \eta}\left[P^{V-1}+P^{u V \phi-1}\right],
\end{aligned}
$$

as we have $V>1$ certainly, from (56). But $u \phi \leq k \phi<1$, whence

$$
\int_{\mathcal{R}\left(q, a_{u}\right)}\left|U\left(\alpha, h_{n}, P^{1 / u}\right)\right|^{u V} d \alpha \ll q^{-V+u V \eta} P^{V-1} \text {. }
$$

By combining with (61), we have

$$
\begin{aligned}
\int_{\mathcal{N}}\left(\prod_{i=1}^{2 t}\left|S_{i}(\alpha)\right|\right)\left|U\left(\alpha, h_{n}, P^{1 / u}\right)\right|^{u V} K(\alpha) d \alpha \\
\ll\left(P_{1} \ldots P_{t}\right)^{2} \sum_{1 \leq q \leq P^{\phi}} \sum_{a_{u} \in \mathbb{Z}} \min \left(1,\left|a_{u}\right|^{-2} q^{2}\right) q^{-V+u V \eta} P^{V-1} \\
\ll\left(P_{1} \ldots P_{t}\right)^{2} P^{V-1} \sum_{1 \leq q \leq P^{\phi}} q^{-V+u V \eta}\left[\sum_{a_{u}=0}^{q} 1+\sum_{l=1}^{\infty} \sum_{a_{u}=l q+1}^{(l+1) q} \frac{1}{l^{2}}\right] \\
\\
\ll\left(P_{1} \ldots P_{t}\right)^{2} P^{V-1} \sum_{1 \leq q \leq P^{\phi}} q^{1-V+u V \eta} \\
\end{aligned}
$$

for sufficiently small $\eta$, since $V>2$ from (56). If we recall that we have already established the bound (53), the proof of Lemma 5 is complete.

5. An analogue of Weyl's inequality. We now proceed to establish an analogue of Weyl's inequality. (For the traditional Weyl inequality, see, for example, Lemma 2.4 of [18].) Our aim is to show, following the method of [2], that there is a function $T(P)$, which tends to infinity as $P$ tends to 
infinity, such that we have

$$
\sup _{P^{\delta-1} \leq|\alpha| \leq T(P)}\left|U_{1}(\alpha, P) U_{2}(\alpha, P)\right|=o\left(P^{1 / w_{1}+1 / w_{2}}\right) .
$$

We now give a lemma which allows us to find good rational approximations to the coefficients of a polynomial if the corresponding exponential sum is large in absolute value. In the following lemma, it is crucial to eliminate extra factors of $N^{\eta}$ that might appear on the right side of the first inequality in (65). When using the ideas of Bentkus and Götze, it is crucial to eliminate this extra factor. Otherwise, we could simply quote Theorem 5.1 of [1]. We note that an observation by Professor Wooley has enabled us to give a much shorter proof. The following proof is based on his ideas, and I am very grateful to him for pointing out the improvement.

LEMMA 6. Suppose that $k$ is a positive integer and that $r(x)$ is a polynomial of degree $k$ with real coefficients. We write

$$
r(x)=\lambda_{k} x^{k}+\lambda_{k-1} x^{k-1}+\ldots+\lambda_{1} x+\lambda_{0} .
$$

Fix any positive number $\eta$. Suppose as well that $N$ is a positive number which is sufficiently large in terms of $k$ and $\eta$. Define the exponential sum

$$
S(N)=\sum_{1 \leq x \leq N} e(r(x)) .
$$

Suppose that $\gamma$ is a positive number for which

$$
|S(N)| \geq \gamma N \text {. }
$$

Then there are positive constants $C_{4}$ and $C_{5}$, each of which depends only on $k$, such that if $\gamma$ satisfies

$$
N^{\eta} \max \left(N^{-2^{1-k}}, N^{-1 /(k+1)}\right) \leq \gamma \leq 1,
$$

then there exists a positive integer $q$ and integers $a_{1}, \ldots, a_{k}$, satisfying $\left(a_{1}, \ldots, a_{k}, q\right)=1$ with

$$
q<C_{4} \gamma^{-k-2 k^{2} \eta}, \quad\left|\lambda_{j}-a_{j} / q\right|<C_{5} \gamma^{-k} N^{-j} \quad \text { for } 1 \leq j \leq k .
$$

We observe that the case $k=1$ of Lemma 6 can be proved easily from the standard estimate

$$
\sum_{1 \leq x \leq N} e\left(\lambda_{1} x\right) \ll \min \left(N,\left\|\lambda_{1}\right\|^{-1}\right) .
$$

In fact, in this case, one can prove a much stronger result, namely that if (63) holds, then $\left\|\lambda_{1}\right\| \ll \gamma^{-1} N^{-1}$. In this case, we can replace (64) with the simple condition that $\gamma$ is a positive number. Thus we no longer consider the case $k=1$.

Proof of Lemma 6. We may clearly assume that $\eta \leq 1 /(2 k)$. As $\gamma \geq N^{-2^{1-k}+\eta}$, we can apply Theorem 5.1 of [1], whence there are integers 
$q, a_{1}, \ldots, a_{k}$ with

$$
\begin{aligned}
& \left(a_{1}, \ldots, a_{k}, q\right)=1, \quad 1 \leq q<\gamma^{-k} N^{\eta}, \\
& \left|\lambda_{j} q-a_{j}\right|<\gamma^{-k} N^{\eta-j} \quad \text { for } 1 \leq j \leq k .
\end{aligned}
$$

For real vectors $\boldsymbol{\beta}=\left(\beta_{1}, \ldots, \beta_{k}\right)$, positive integers $q$ and integral vectors $\mathbf{a}=\left(a_{1}, \ldots, a_{k}\right)$, define

$$
\begin{aligned}
I(\boldsymbol{\beta}) & =\int_{0}^{N} e\left(\beta_{1} \gamma+\beta_{2} \gamma^{2}+\ldots+\beta_{k} \gamma^{k}\right) d \gamma, \\
S(q, \mathbf{a}) & =\sum_{x=1}^{q} e\left(\frac{a_{1} x+a_{2} x^{2}+\ldots+a_{k} x^{k}}{q}\right) .
\end{aligned}
$$

Then, if we write $\beta_{j}=\lambda_{j}-a_{j} / q$ for $1 \leq j \leq k$, by Theorem 7.2 of [18], it follows that

$$
S(N)=q^{-1} S(q, \mathbf{a}) I(\boldsymbol{\beta})+O\left(q\left(1+\sum_{j=1}^{k}\left|\beta_{j}\right| N^{j}\right)\right) .
$$

By (66), it follows that

$$
S(N)=q^{-1} S(q, \mathbf{a}) I(\boldsymbol{\beta})+O\left(\gamma^{-k} N^{\eta}\right) .
$$

As $\gamma \geq N^{\eta-1 /(k+1)}$, for large $N$ we must have

$$
\gamma N \leq|S(N)| \leq\left|q^{-1} S(q, \mathbf{a}) I(\boldsymbol{\beta})\right| .
$$

By Theorems 7.1 and 7.3 of [18],

$$
\gamma N \ll q^{\eta-1 / k} N\left(1+\sum_{j=1}^{k}\left|\lambda_{j}-\frac{a_{j}}{q}\right| N^{j}\right)^{-1 / k},
$$

whence

$$
q^{1 / k-\eta} \ll \gamma^{-1}, \quad\left|\lambda_{j}-a_{j} / q\right| N^{j} \ll \gamma^{-k} \quad \text { for } 1 \leq j \leq k .
$$

The proof of Lemma 6 follows.

From this point onward, we follow [13] quite closely, which we note was in turn motivated by the work of Bentkus and Götze [2]. We have the following lemma, which is similar to Lemma 3 of [13] and to Theorem 6.1 of [2]. The proof follows that of Lemma 3 of [13] quite closely, so we omit it.

Lemma 7. Suppose that we are in the setting of Proposition 1. Suppose that $T_{0}$ and $T$ are real numbers with $0<T_{0} \leq 1 \leq T$. Then

$$
\sup _{T_{0} \leq|\alpha| \leq T}\left|U_{1}(\alpha, P) U_{2}(\alpha, P)\right|=o\left(P^{1 / w_{1}+1 / w_{2}}\right) .
$$

Now we give a result which is an almost exact analogue of Lemma 4 of [13]. The present lemma follows in much the same manner as Lemma 4 of [13], so we omit its proof. 
Lemma 8. Suppose that we are in the setting of Proposition 1. Then there are positive real-valued functions $T_{0}(P)$ and $T(P)$, depending only on the coefficients $\mu_{1 j_{1}}$ and $\mu_{2 j_{2}}$, for which

$$
\lim _{P \rightarrow \infty} T_{0}(P)=0 \quad \text { and } \quad \lim _{P \rightarrow \infty} T(P)=\infty,
$$

and so that

$$
P^{-\delta} \leq T_{0}(P) \leq 1 \quad \text { for } P \geq 3
$$

and

$$
\sup _{T_{0}(P) \leq|\alpha| \leq T(P)}\left|U_{1}(\alpha, P) U_{2}(\alpha, P)\right|=o\left(P^{1 / w_{1}+1 / w_{2}}\right) .
$$

This completes our analogue of Weyl's inequality for one part of the minor arcs. We note that now we have chosen the function $T(P)$ which is used to define the minor arcs.

Now we need to handle the remaining, easier, region of the minor arcs. The following lemma is very similar to Lemma 5 of [13], and is proved in much the same manner. We omit the proof.

Lemma 9. Suppose that we are in the setting of Proposition 1. Then

$$
\sup _{P^{\delta-1} \leq|\alpha| \leq T_{0}(P)}\left|U_{1}(\alpha, P)\right|=o\left(P^{1 / w_{1}}\right) .
$$

6. The minor arcs and trivial arcs. We first apply our analogues of Hua's inequality and Weyl's inequality to treat the minor arcs. We have the following lemma.

Lemma 10. Suppose that we are in the setting of Proposition 1 , and that the functions $S_{i}(\alpha)$ and $U_{n}(\alpha)$ and $K(\alpha)$ are defined as above. Then

$$
\int_{\mathfrak{m}}\left(\prod_{i=1}^{2 t}\left|S_{i}(\alpha)\right|\right)\left(\prod_{n=1}^{m}\left|U_{n}(\alpha)\right|\right) K(\alpha) d \alpha=o\left(P^{W-1}\left(P_{1} \ldots P_{t}\right)^{2}\right)
$$

Proof. Observe first that

$$
\begin{aligned}
& \int_{\mathfrak{m}}\left(\prod_{i=1}^{2 t}\left|S_{i}(\alpha)\right|\right)\left(\prod_{n=1}^{m}\left|U_{n}(\alpha)\right|\right) K(\alpha) d \alpha \\
& \ll\left(\sup _{\alpha \in \mathfrak{m}}\left|U_{1}(\alpha, P) U_{2}(\alpha, P)\right|\right) \int_{\mathbb{R}}\left(\prod_{i=1}^{2 t}\left|S_{i}(\alpha)\right|\right)\left(\prod_{n=3}^{m}\left|U_{n}(\alpha)\right|\right) K(\alpha) d \alpha .
\end{aligned}
$$

The integral on the right side of $(70)$ is $O\left(\left(P_{1} \ldots P_{t}\right)^{2} P^{W-1 / w_{1}-1 / w_{2}-1}\right)$, by Lemma 5 . On the other hand, by applying Lemmas 8 and 9 , and recalling the definition (40) of $\mathfrak{m}$, we have

$$
\sup _{\alpha \in \mathfrak{m}}\left|U_{1}(\alpha, P) U_{2}(\alpha, P)\right|=o\left(P^{1 / w_{1}+1 / w_{2}}\right) .
$$

Inserting these two observations in the bound (70) proves Lemma 10. 
At this point, we treat the contribution to the integral (37) from the trivial arcs. We first must state a slight generalization of a lemma due to Davenport and Roth. (See Lemma 2 of [10].)

Lemma 11 (Davenport and Roth). Fix a positive number $\eta$. Let

$$
F(\alpha)=\sum e\left(\alpha f\left(x_{1}, \ldots, x_{s}\right)\right),
$$

where $f$ is any real function of $s$ variables, and the summation is over any finite set of values of $x_{1}, \ldots, x_{s}$. Define the function $L$ by

$$
L(\alpha)=\frac{(\sin (\pi \eta \alpha))^{2}}{\pi^{2} \alpha^{2} \eta} .
$$

Then for any real number $A$ with $A>4 / \eta$, we have

$$
\int_{|\alpha|>A}|F(\alpha)|^{2} L(\alpha) d \alpha \leq \frac{16}{\eta A} \int_{\mathbb{R}}|F(\alpha)|^{2} L(\alpha) d \alpha .
$$

We observe that in the case $\eta=1$, the lemma is exactly Lemma 2 of [10]. The proof of the general case can be deduced easily from the case $\eta=1$ by a change of variable, so we omit the details.

Now we can complete the treatment of the trivial arcs. We have the following result.

Lemma 12. Suppose that we are in the setting of Proposition 1, and that the functions $S_{i}(\alpha)$ and $U_{n}(\alpha)$ and $K(\alpha)$ are defined as above. Then

$$
\int_{\mathfrak{t}}\left(\prod_{i=1}^{2 t}\left|S_{i}(\alpha)\right|\right)\left(\prod_{n=1}^{m}\left|U_{n}(\alpha)\right|\right) K(\alpha) d \alpha=o\left(P^{W-1}\left(P_{1} \ldots P_{t}\right)^{2}\right) .
$$

Proof. Recall from the statement of Proposition 1 that $m=2 k+4$. Thus we may use Hölder's inequality and the fact that $K(\alpha)$ is positive to see that the left hand side of (71) is

$$
\begin{aligned}
\ll & \left.\int_{|\alpha|>T(P)}\left(\prod_{i=1}^{t}\left|S_{i}(\alpha)\right|^{2}\right)\left(\prod_{n=1}^{m / 2}\left|U_{n}(\alpha)\right|^{2}\right) K(\alpha) d \alpha\right)^{1 / 2} \\
& \times\left(\int_{|\alpha|>T(P)}\left(\prod_{i=t+1}^{2 t}\left|S_{i}(\alpha)\right|^{2}\right)\left(\prod_{n=m / 2+1}^{m}\left|U_{n}(\alpha)\right|^{2}\right) K(\alpha) d \alpha\right)^{1 / 2} .
\end{aligned}
$$

We will show only that

$$
\begin{aligned}
\int_{|\alpha|>T(P)}\left(\prod_{i=1}^{t}\left|S_{i}(\alpha)\right|^{2}\right)\left(\prod_{n=1}^{m / 2}\left|U_{n}(\alpha)\right|^{2}\right) K(\alpha) d \alpha \\
=o\left(P^{\left(\sum_{n=1}^{m / 2} 2 / w_{n}\right)-1}\left(P_{1} \ldots P_{t}\right)^{2}\right) .
\end{aligned}
$$


One can show the corresponding bound

$$
\begin{array}{r}
\int_{|\alpha|>T(P)}\left(\prod_{i=t+1}^{2 t}\left|S_{i}(\alpha)\right|^{2}\right)\left(\prod_{n=m / 2+1}^{m}\left|U_{n}(\alpha)\right|^{2}\right) K(\alpha) d \alpha \\
=o\left(P^{\left(\sum_{n=m / 2+1}^{m} 2 / w_{n}\right)-1}\left(P_{1} \ldots P_{t}\right)^{2}\right)
\end{array}
$$

for the other integral in (72) in a very similar fashion, so we omit that part of the proof.

Note that our choice of $K(\alpha)$, made using Lemma 1, is of course exactly the function $L(\alpha)$ of Lemma 11 with $\eta=\varepsilon$. Thus, for sufficiently large $P$, if we recall that $T(P)$ tends to infinity as $P$ tends to infinity, Lemma 11 yields

$$
\begin{aligned}
\int_{|\alpha|>T(P)}\left(\prod_{i=1}^{t}\left|S_{i}(\alpha)\right|^{2}\right) & \left(\prod_{n=1}^{m / 2}\left|U_{n}(\alpha)\right|^{2}\right) K(\alpha) d \alpha \\
& \ll \frac{1}{T(P)} \int_{\mathbb{R}}\left(\prod_{i=1}^{t}\left|S_{i}(\alpha)\right|^{2}\right)\left(\prod_{n=1}^{m / 2}\left|U_{n}(\alpha)\right|^{2}\right) K(\alpha) d \alpha .
\end{aligned}
$$

Combining this bound with Lemma 5 yields

$$
\begin{aligned}
\int_{|\alpha|>T(P)}\left(\prod_{i=1}^{2 t}\left|S_{i}(\alpha)\right|^{2}\right)\left(\prod_{n=1}^{m / 2}\left|U_{n}(\alpha)\right|^{2}\right) & K(\alpha) d \alpha \\
& \ll \frac{1}{T(P)}\left(P_{1} \ldots P_{t}\right)^{2} P^{\left(\sum_{n=1}^{m / 2} 2 / w_{n}\right)-1} ;
\end{aligned}
$$

observe that here we have used the special case of Lemma 5 in which $\lambda_{(t+i) j}=\lambda_{i j}$ for $1 \leq i \leq t$ and $1 \leq j \leq v_{i}$. But $T(P)$ was chosen in Lemma 8 to satisfy $\lim _{P \rightarrow \infty} T(P)=\infty$, whence the bound (73) follows. Therefore the proof of Lemma 12 is complete.

7. The major arc. We now come to the contribution of the major arc to the integral (37). We show that

$$
\int_{\mathcal{M}}\left(\prod_{i=1}^{2 t} S_{i}(\alpha)\right)\left(\prod_{n=1}^{m} U_{n}(\alpha)\right) e(-M \alpha) K(\alpha) d \alpha \gg\left(P_{1} \ldots P_{t}\right)^{2} P^{W-1}
$$

for sufficiently large $P$. This will complete the proof of Proposition 1 , in view of Lemmas 10 and 12 . The following treatment is largely very standard, but we give the proof for the sake of completeness.

We start off by applying Lemma 4 to the generating functions $U_{n}(\alpha)$. For $\alpha \in \mathcal{M}$, we have $|\alpha| \leq P^{\delta-1}$. From (38), we have $\delta<1 / k \leq 1 / w_{n}$. Thus 
for $\alpha \in \mathcal{M}$, for $1 \leq n \leq m$, and for large $P$, we certainly have

$$
\left|\alpha \mu_{n j}\right| \leq \frac{P^{(1-j) / w_{n}}}{2 w_{n}^{2}} \quad \text { for } 1 \leq j \leq w_{n} .
$$

Therefore, for $\alpha \in \mathcal{M}$ and $1 \leq n \leq m$, Lemma 4 yields

$$
U_{n}(\alpha)=I_{n}(\alpha)+O(1),
$$

where

$$
I_{n}(\alpha)=\int_{0}^{P^{1 / w_{n}}} e\left(\sum_{j=1}^{w_{n}} \alpha \mu_{n j} x^{j}\right) d x .
$$

It follows by a telescoping series argument that for $\alpha \in \mathcal{M}$,

$$
\prod_{n=1}^{m} U_{n}(\alpha)=\prod_{n=1}^{m} I_{n}(\alpha)+O\left(P^{W-1 / k}\right) .
$$

Using this approximation, trivial estimates and the bound (26), we obtain

$$
\begin{aligned}
\int_{\mathcal{M}}\left(\prod_{i=1}^{2 t} S_{i}(\alpha)\right)\left(\prod_{n=1}^{m} U_{n}(\alpha)\right) e(-M \alpha) K(\alpha) d \alpha \\
=\int_{\mathcal{M}}\left(\prod_{i=1}^{2 t} S_{i}(\alpha)\right)\left(\prod_{n=1}^{m} I_{n}(\alpha)\right) e(-M \alpha) K(\alpha) d \alpha \\
\quad+O\left(\left(P_{1} \ldots P_{t}\right)^{2} P^{W-1+\delta-1 / k}\right) .
\end{aligned}
$$

But now, using (26), trivial estimates and Theorem 7.3 of [18], we also have

$$
\begin{aligned}
\int_{\mathbb{R} \backslash \mathcal{M}}\left(\prod_{i=1}^{2 t} S_{i}(\alpha)\right)\left(\prod_{n=1}^{m} I_{n}(\alpha)\right) e(-M \alpha) K(\alpha) d \alpha & \\
& \ll\left(P_{1} \ldots P_{t}\right)^{2} \int_{\mathbb{R} \backslash \mathcal{M}} \prod_{n=1}^{m}\left|\mu_{n w_{n}} \alpha\right|^{-1 / w_{n}} d \alpha \\
& \ll\left(P_{1} \ldots P_{t}\right)^{2}\left(P^{\delta-1}\right)^{1-W} \\
& \ll\left(P_{1} \ldots P_{t}\right)^{2} P^{W-1} P^{\delta(1-W)} ;
\end{aligned}
$$

here we have used the fact that $W>1$, which clearly follows from (36).

Combining (75) and (76) and using (38), we have

$$
\begin{aligned}
\int_{\mathcal{M}} & \left(\prod_{i=1}^{2 t} S_{i}(\alpha)\right)\left(\prod_{n=1}^{m} U_{n}(\alpha)\right) e(-M \alpha) K(\alpha) d \alpha \\
& =\int_{\mathbb{R}}\left(\prod_{i=1}^{2 t} S_{i}(\alpha)\right)\left(\prod_{n=1}^{m} I_{n}(\alpha)\right) e(-M \alpha) K(\alpha) d \alpha+o\left(\left(P_{1} \ldots P_{t}\right)^{2} P^{W-1}\right) .
\end{aligned}
$$


Thus to prove that (74) holds for large $P$, it suffices to show that for large $P$ we have

$$
\int_{\mathbb{R}}\left(\prod_{i=1}^{2 t} S_{i}(\alpha)\right)\left(\prod_{n=1}^{m} I_{n}(\alpha)\right) e(-M \alpha) K(\alpha) d \alpha \gg\left(P_{1} \ldots P_{t}\right)^{2} P^{W-1} .
$$

Now, by (26) and trivial estimates, the integral on the left side of (77) is absolutely convergent. Thus we may rewrite this integral as

$$
\begin{aligned}
\sum_{P_{1} \leq x_{1}, x_{t+1} \leq 2 P_{1}} \ldots \sum_{P_{t} \leq x_{t}, x_{2 t} \leq 2 P_{t}} \int_{\prod_{n=1}^{m}\left[0, P^{1 / w_{n}}\right]} \int_{\mathbb{R}} \\
e\left(\alpha\left(f_{1}\left(x_{1}\right)+\ldots+f_{2 t}\left(x_{2 t}\right)+g_{1}\left(y_{1}\right)+\ldots+g_{m}\left(y_{m}\right)-M\right)\right) K(\alpha) d \alpha d \mathbf{y} .
\end{aligned}
$$

Therefore, by the identity (25), we may see that the integral on the left side of (77) is bounded below by

$$
\frac{1}{2} \sum_{P_{1} \leq x_{1}, x_{t+1} \leq 2 P_{1}} \cdots \sum_{P_{t} \leq x_{t}, x_{2 t} \leq 2 P_{t}} \int_{\mathcal{R}} d \mathbf{y},
$$

where one defines $\mathcal{R}=\mathcal{R}\left(x_{1}, \ldots, x_{2 t}\right)$ to be the set

$$
\left\{\mathbf{y} \in \prod_{n=1}^{m}\left[0, P^{1 / w_{n}}\right]:\left|\sum_{i=1}^{2 t} f_{i}\left(x_{i}\right)+\sum_{n=1}^{m} g_{n}\left(y_{n}\right)-M\right|<\frac{\varepsilon}{2}\right\} .
$$

So to prove (77), it is enough to show that for all choices of (2t)-tuples $\left(x_{1}, \ldots, x_{2 t}\right)$ with $P_{i} \leq x_{i}, x_{t+i} \leq 2 P_{i}$ for $1 \leq i \leq t$,

$$
\mu\left(\mathcal{R}\left(x_{1}, \ldots, x_{2 t}\right)\right) \gg P^{W-1},
$$

where $\mu$ denotes $m$-dimensional measure. We turn now to proving the bound (78) in each case of Proposition 1.

Consider case (i) of Proposition 1. Recall that we have assumed that condition (14) holds, namely that

$$
\sum_{i=1}^{2 t} \sum_{j=1}^{v_{i}}\left|\lambda_{i j}\right|+\sum_{n=3}^{m} \sum_{j=1}^{w_{n}}\left|\mu_{n j}\right| \leq \frac{\mu_{1 w_{1}}}{2^{k+3}} .
$$

Recall as well from (29) that $P_{i}^{v_{i}} \leq P$ for $1 \leq i \leq t$. It follows that given any real vectors $\mathbf{x} \in \mathbb{R}^{2 t}$ and $\mathbf{y}=\left(y_{2}, \ldots, y_{m}\right) \in \mathbb{R}^{m-1}$ with $P_{i} \leq x_{i}, x_{t+i} \leq 2 P_{i}$ for $1 \leq i \leq t$ and with $0 \leq y_{n} \leq P^{1 / w_{n}}$ for $2 \leq n \leq m$, for sufficiently large $P$ we have

$$
\left|\sum_{i=1}^{2 t} f_{i}\left(x_{i}\right)+\sum_{n=3}^{m} g_{n}\left(y_{n}\right)+\sum_{j=1}^{w_{2}-1} \mu_{2 j} y_{2}^{j}\right| \leq \frac{\mu_{1 w_{1} P}}{8} .
$$

Now fix any integer vector $\mathbf{x} \in \mathbb{Z}^{2 t}$ and real vector $\left(y_{3}, \ldots, y_{m}\right)$ with

$$
P_{i} \leq x_{i}, x_{t+i} \leq 2 P_{i} \quad \text { for } 1 \leq i \leq t, \quad 0 \leq y_{n} \leq P^{1 / w_{n}} \quad \text { for } 3 \leq n \leq m .
$$


Then consider any real number $y_{2}$ satisfying

$$
(7 / 12)^{1 / w_{2}} P^{1 / w_{2}} \leq y_{2} \leq(2 / 3)^{1 / w_{2}} P^{1 / w_{2}} .
$$

Recall that we have assumed in case (i) of Proposition 1 that $\mu_{2 w_{2}}$ is negative. Thus we have

$$
-\frac{7}{12} \mu_{2 w_{2}} P \leq-\mu_{2 w_{2}} y_{2}^{w_{2}} \leq-\frac{2}{3} \mu_{2 w_{2}} P .
$$

Recall also the condition $1 \leq\left|\mu_{1 w_{1}} / \mu_{2 w_{2}}\right| \leq 2$ from case (i) of the proposition. It follows that

$$
\frac{7}{24} \mu_{1 w_{1}} P \leq-\mu_{2 w_{2}} y_{2}^{w_{2}} \leq \frac{2}{3} \mu_{1 w_{1}} P .
$$

Also, recall from (15) that we have assumed $|M| \leq\left(\mu_{1 w_{1}} P\right) / 8$, whence from (79) we have

$$
\frac{1}{24} \mu_{1 w_{1}} P \leq\left(M-g_{2}\left(y_{2}\right)-\sum_{i=1}^{2 t} f_{i}\left(x_{i}\right)-\sum_{n=3}^{m} g_{n}\left(y_{n}\right)\right) \leq \frac{11}{12} \mu_{1 w_{1}} P .
$$

It follows for large $P$ that there is a real number $y_{1}$ with

$$
(1 / 25)^{1 / w_{1}} P^{1 / w_{1}} \leq y_{1} \leq(12 / 13)^{1 / w_{1}} P^{1 / w_{1}}
$$

which satisfies

$$
g_{1}\left(y_{1}\right)=M-g_{2}\left(y_{2}\right)-\sum_{i=1}^{2 t} f_{i}\left(x_{i}\right)-\sum_{n=3}^{m} g_{n}\left(y_{n}\right) .
$$

Now, for this choice of $y_{1}$ and any real number $L$ with $|L| \leq 1$, and for sufficiently large $P$, we have

$$
\left|g_{1}\left(y_{1}+L\right)-g_{1}\left(y_{1}\right)\right| \ll L P^{1-1 / w_{1}} .
$$

Thus it follows that for each choice as above of $\left(x_{1}, \ldots, x_{2 t}, y_{2}, \ldots, y_{n}\right)$, there is an interval $\mathcal{I} \subseteq\left[0, P^{1 / w_{1}}\right]$ of choices $y_{1}$ of length $\gg_{\varepsilon} P^{1 / w_{1}-1}$ for which

$$
\left|\sum_{i=1}^{2 t} f_{i}\left(x_{i}\right)+\sum_{n=1}^{m} g_{n}\left(y_{n}\right)-M\right|<\frac{\varepsilon}{2}
$$

Thus for each fixed choice of $\left(x_{1}, \ldots, x_{2 t}\right)$ as above, there is a set of $m$ dimensional measure

$$
\gg_{\varepsilon} P^{1 / w_{1}-1} P^{1 / w_{2}} \prod_{n=3}^{m} P^{1 / w_{n}} \gg_{\varepsilon} P^{W-1}
$$

which is contained in $\mathcal{R}$. This completes our proof in case (i). 
The proof of case (ii) of Proposition 1 is similar, and in fact slightly more simple, so we omit it. In both cases of Proposition 1, it follows that the contribution to the integral (37) from the major arc is $\gg\left(P_{1} \ldots P_{t}\right)^{2} P^{W-1}$. As noted above, this is enough to complete the proof of Proposition 1. As also observed above, this completes the proof of Theorems 1 and 2 .

\section{References}

[1] R. C. Baker, Diophantine Inequalities, London Math. Soc. Monographs (N.S.) 1, Oxford Univ. Press, New York, 1986.

[2] V. Bentkus and F. Götze, Lattice point problems and distribution of values of quadratic forms, Ann. of Math. (2) 150 (1999), 977-1027.

[3] —, - Lattice points in multidimensional bodies, Forum Math. 13 (2001), 149-225.

[4] B. J. Birch, Homogeneous forms of odd degree in a large number of variables, Mathematika 4 (1957), 102-105.

[5] J. Brüdern, The Davenport-Heilbronn Fourier transform method, and some Diophantine inequalities, in: Number Theory and its Applications (Kyoto, 1997), Dev. Math. 2, Kluwer, Dordrecht, 1999, 59-87.

[6] J. Brüdern and R. J. Cook, On simultaneous diagonal equations and inequalities, Acta Arith. 62 (1992), 125-149.

[7] R. J. Cook and S. Raghavan, On positive definite quadratic polynomials, ibid. 45 (1986), 319-328.

[8] H. Davenport and H. Heilbronn, On indefinite quadratic forms in five variables, J. London Math. Soc. 21 (1946), 185-193.

[9] H. Davenport and D. J. Lewis, Non-homogeneous cubic equations, ibid. 39 (1964), 657-671.

[10] H. Davenport and K. F. Roth, The solubility of certain Diophantine inequalities, Mathematika 2 (1955), 81-96.

[11] K. Ford, The representation of numbers as sums of unlike powers. II, J. Amer. Math. Soc. 9 (1996), 919-940.

[12] —, Waring's problem with polynomial summands, J. London Math. Soc. (2) 61 (2000), 671-680.

[13] D. E. Freeman, Asymptotic lower bounds for Diophantine inequalities, Mathematika, to appear.

[14] E. Kamke, Verallgemeinerungen des Waring-Hilbertschen Satzes, Math. Ann. 83 (1921), 85-112.

[15] M. Nathanson, Elementary Methods in Number Theory, Springer, New York, 2000.

[16] W. M. Schmidt, Diophantine inequalities for forms of odd degree, Adv. Math. 38 (1980), 128-151.

[17] - The density of integer points on homogeneous varieties, Acta Math. 154 (1985), 243-296.

[18] R. C. Vaughan, The Hardy-Littlewood Method, 2nd ed., Cambridge Univ. Press, Cambridge, 1997.

[19] G. L. Watson, Indefinite quadratic polynomials, Mathematika 7 (1960), 141-144.

[20] —, Quadratic Diophantine equations, Philos. Trans. Roy. Soc. London Ser. A 253 (1960), 227-254.

[21] H. Weyl, Über die Gleichverteilung von Zahlen mod. Eins, Math. Ann. 77 (1916), 313-352. 
[22] T. D. Wooley, On Vinogradov's mean value theorem, Mathematika 39 (1992), 379-399.

[23] - On exponential sums over smooth numbers, J. Reine Angew. Math. 488 (1997), 79-140.

Department of Mathematics

University of Colorado

395 UCB

Boulder, CO 80309-0395, U.S.A.

Current address:

School of Mathematics

Institute for Advanced Study

1 Einstein Drive

Princeton, NJ 08540, U.S.A.

E-mail: freem@ias.edu

Received on 20.11.2001

$(4154)$ 